Review Article

\title{
Helicobacter pylori Infection Is Associated with Type 2 Diabetes, Not Type 1 Diabetes: An Updated Meta-Analysis
}

\author{
Jun-Zhen Li, Jie-Yao Li, Ting-Feng Wu, Ji-Hao Xu, Can-Ze Huang, Di Cheng, \\ Qi-Kui Chen, and Tao Yu
}

\begin{abstract}
Department of Gastroenterology, Sun Yat-Sen Memorial Hospital, Sun Yat-Sen University, 107 Yan Jiang Xi Road, 510120 Guangzhou, Guangdong, China
\end{abstract}

Correspondence should be addressed to Qi-Kui Chen; qkchen2015@163.com and Tao Yu; yutao2014@126.com

Received 9 January 2017; Accepted 14 May 2017; Published 13 August 2017

Academic Editor: Paul Enck

Copyright (C) 2017 Jun-Zhen Li et al. This is an open access article distributed under the Creative Commons Attribution License, which permits unrestricted use, distribution, and reproduction in any medium, provided the original work is properly cited.

\begin{abstract}
Background. Extragastric manifestations of Helicobacter pylori (H. pylori) infection have been reported in many diseases. However, there are still controversies about whether $H$. pylori infection is associated with diabetes mellitus (DM). This study was aimed at answering the question. Methods. A systematic search of the literature from January 1996 to January 2016 was conducted in PubMed, Embase databases, Cochrane Library, Google Scholar, Wanfang Data, China national knowledge database, and SinoMed. Published studies reporting H. pylori infection in both DM and non-DM individuals were recruited. Results. 79 studies with 57,397 individuals were included in this meta-analysis. The prevalence of $H$. pylori infection in DM group (54.9\%) was significantly higher than that $(47.5 \%)$ in non-DM group $(\mathrm{OR}=1.69, P<0.001)$. The difference was significant in comparison between type $2 \mathrm{DM}$ group and non-DM group $(\mathrm{OR}=2.05)$, but not in that between type $1 \mathrm{DM}$ group and non-DM group $(\mathrm{OR}=1.23,95 \% \mathrm{CI}: 0.77-1.96, \mathrm{P}=0.38)$. Conclusion. Our meta-analysis suggested that there is significantly higher prevalence of $H$. pylori infection in DM patients as compared to non-DM individuals. And the difference is associated with type $2 \mathrm{DM}$ but not type $1 \mathrm{DM}$.
\end{abstract}

\section{Introduction}

Helicobacter pylori (H. pylori) is a gram-negative spiral bacterium, colonized in the stomach. Approximately one-half of the population over the world is infected with $H$. pylori [1]. Many researches have proved that $H$. pylori infection is highly associated with gastrointestinal diseases such as chronic gastritis, peptic ulcer disease, gastric cancer, and mucosa-associated lymphoid tissue (MALT) lymphoma since its discovery [2]. In addition, extragastric disorders associated with $H$. pylori infection, such as cardiovascular diseases and metabolic syndrome, have been revealed and some of them were characterized by persistent and lowgrade systemic inflammation [3]. Inflammation has been demonstrated to play an important part in the pathogenesis of diabetes mellitus (DM), especially type 2 DM (T2DM) [4]. On the other hand, Kondrashova and Hyöty reviewed that some microbes served as the risk factor participating in the trigger and the development of type 1 DM (T1DM), but some microbes such as $H$. pylori served as a protective factor by lowering the risk of T1DM [5]. Above all, $H$. pylori infection was a factor not negligible in the process of DM.

Since Simon et al. firstly reported the association between $H$. pylori infection and DM [6], many studies were carried out. Several case-control studies have reported a higher prevalence of $H$. pylori infection in DM patients $[7,8]$. Some cross-sectional researches also revealed a significant correlation between $H$. pylori infection and diabetes [9-11]. Moreover, a meta-analysis carried out by Zhou et al. suggested a trend toward more frequent $H$. pylori infection in DM patients, especially in T2DM patients [12]. However, Tamura et al. found a significantly higher DM prevalence among individuals with $H$. pylori infection than those without, but the 
difference could be mostly ascribed to older age [13]. And some studies argued that no difference in the prevalence of $H$. pylori infection was found between DM and nonDM individuals $[14,15]$. Overall, this subject remains controversial now.

The present updated meta-analysis was conducted to answer if there is a difference in the prevalence of $H$. pylori infection between DM and non-DM individuals. Subgroup analyses were carried out based on the types of DM, geographical regions, and methods for $H$. pylori detection to further investigate the relationship between $H$. pylori infection and DM.

\section{Methods}

2.1. Search Strategy and Selection Criteria. Published guidelines for conducting meta-analyses were followed [16]. We searched PubMed, Embase databases, Cochrane Library, Google Scholar, Wanfang Data (Chinese), China national knowledge database (Chinese), and SinoMed (Chinese) for all relevant articles reported from January 1996 to January 2016, with combinations of the search terms "Helicobacter pylori," or " $H$. pylori," or "Campylobacter pylori," or "C. pylori," and "diabetes mellitus," or "diabetes," or "type 1 diabetes," or "type 1 diabetes mellitus," or "type 2 diabetes" or "type 2 diabetes mellitus".

To be eligible for inclusion, studies had to meet the following criteria: (1) they were published studies which reported $H$. pylori infection in DM individuals and nonDM individuals (individuals without DM, impaired glucose tolerance, or impaired fasting glucose); (2) detailed data of $H$. pylori infection rate in both groups was provided. Studies that did not meet the inclusion criteria were not enrolled.

Studies were excluded if they were as follows: (1) duplicate publications; (2) case report, review, meta-analysis, or guideline; (3) not reporting clinically relevant outcomes; and (4) not providing enough details.

2.2. Data Extraction and Quality Assessment. Data were extracted by one investigator, verified by another investigator, and recorded in a well-designed form developed for this study. The data items included authors, year of publication, country, study design, methods of $H$. pylori detection, strains of $H$. pylori, types of DM, age, and sample size. The Newcastle-Ottawa scale (NOS) scoring system was used to assess the quality of the studies [17].

2.3. Statistical Analysis. To obtain pooled effect estimates, the random effects model or fixed effects model was used for meta-analysis, according to the heterogeneity among studies. If there was no statistically significant heterogeneity (twotailed $P$ value $>0.05$ ) among the pooled studies, the fixed effect model would be applied; otherwise, the random effect model would be applied [18]. Odds ratio (OR) with 95\% confidence interval (CI) was used for the case-control and crosssectional studies, while risk ratio (RR) was for the cohort studies. The presence of between-study heterogeneity was estimated using Q-test and $I^{2}$ statistics. Sources of heterogeneity were explored by conducting subgroup analyses based on types of DM, geographical regions, and methods of $H$. pylori detection. The two-sided tests with significance level of 0.05 were conducted in pooled analyses and subgroup analyses using RevMan software (Version 5.3 for Windows, Cochrane Collaboration, Oxford, UK). Publication bias was evaluated graphically by the funnel plots and statistically by Begg's test and Egger's test with the STATA software (Version 14.0; STATA Corporation, College Station, TX, US). $P r$ and $P$ value less than 0.05 were considered representative of no statistically significant publication bias. If publication bias was indicated, the trim and fill method procedure was performed to identify and correct the publication bias [19]. The basis of the method was to (1) "trim" (remove) the studies causing funnel plot asymmetry, (2) use the trimmed funnel plot to estimate the true "centre" of the funnel, and then (3) replace the removed studies and their missing "counterparts" around the centre (filling). An estimate of the number of missing studies was provided; an adjusted OR is derived by performing a meta-analysis including the filled studies.

\section{Results}

3.1. Description of Studies. A total of 783 studies were retrieved from PubMed, Embase databases, Cochrane Library, Google Scholar, Wanfang Data (Chinese), China national knowledge database (Chinese), and SinoMed (Chinese). According to the criteria for inclusion and exclusion, 79 studies were included in this meta-analysis (Figure 1). The included study characteristics were summarized in Table 1. All of the articles were qualified to be pooled with quality score of NOS over 5.76 studies were either casecontrol or cross-sectional studies, and 3 were prospective cohort ones.

A total of 57,397 individuals were enrolled in these studies, with a total $H$. pylori infection prevalence of $49.7 \%$ $(28,542 / 57,397)$. The pooled $H$. pylori infection rate was $54.9 \%(9434 / 17,187)$ in DM group and $47.5 \%(19,108 /$ 40,210 ) in non-DM group. The OR was 1.69 (95\% CI: $1.47-$ $1.95, P<0.001)$ for the two groups. There was high heterogeneity among the studies $\left(I^{2}=86 \%\right)$. The forest plot for pooled prevalence is showed in Figure 2. Each study was sequentially removed from the analysis, and the adjusted ORs (1.63-1.73) were approximate to the initial ones. Especially, the study of Han et al. [20] recruited a total of 6395 patients in DM group and 24,415 in non-DM group, which accounted for nearly one-third of the enrolled individuals in this analysis. However, after removing the data of $\mathrm{Han}$ et al. and re-analyzing, the adjusted odds $(\mathrm{OR}=1.71)$ and heterogeneity $\left(I^{2}=83 \%\right)$ were still approximate to the initial ones in spite of its overweight scale.

3.2. Subgroup Analysis. We found a significant association between $H$. pylori infection and DM but the pooled analysis was with high heterogeneity $\left(I^{2}=86 \%\right)$. Subgroup analyses based on the types of DM, geographical regions, and methods for $H$. pylori detection were conducted to detect the sources of heterogeneity. 


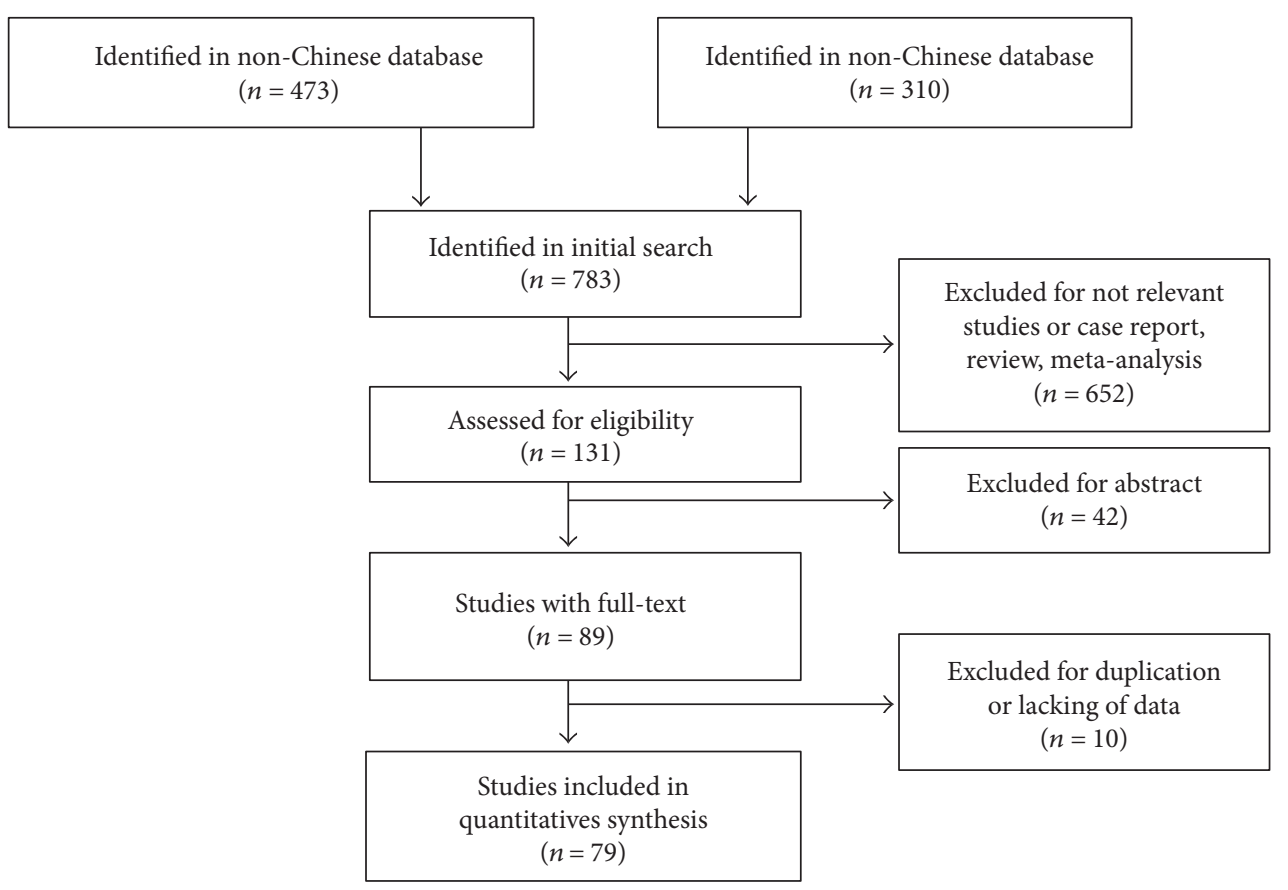

Figure 1: Flow diagram of study selection.

\section{(1) Types of DM}

12 studies with 3175 individuals were assigned to the T1DM subgroup, while 42 studies with 41,684 individuals were to the T2DM subgroup. No significant difference was found between T1DM group and non-DM group in $H$. pylori infection rate $(\mathrm{OR}=1.23,95 \% \mathrm{CI}$ : $0.77-1.96$, $P=0.38$; Figure 3 ). On the contrary, the pooled data indicated that the prevalence of $H$. pylori infection in T2DM was significantly higher than that in non-DM group $(\mathrm{OR}=2.05,95 \% \mathrm{CI}: 1.67-2.52, P<0.001$; Figure 3). Each study including the study by $\mathrm{Han}$ et al. with overweight scale was sequentially removed in the subgroups and the adjusted ORs (1.93-2.10 in T2DM and 1.10-1.42 in T1DM) approximated to the initial ones.

\section{(2) Geographical regions}

Subgroup studies stratified by geographical regions were performed. The recruited individuals were mostly from Asia $(75.8 \%, 43,523 / 57,397)$. The infection rate was $51.7 \%$ $(22,503 / 43,523), 39.7 \%$ (2969/7479), 47.3\% (2562/5411), and 48.7\% (499/1024) in group Asia, group Europe, group America, and group Africa, respectively. No significant difference of $H$. pylori infection rate between DM and nonDM individuals was found in group America and group Africa ( $P=0.36$ for America; $P=0.38$ for Africa). However, in group Asia and group Europe, significantly higher $H$. pylori infection rate was detected in DM individuals $(\mathrm{OR}=2.04$ and $\mathrm{OR}=1.40$, resp.). But there was still high heterogeneity within these subgroups $\left(I^{2}=68 \%-90 \%\right.$; Figure 4$)$.
(3) Methods for H. pylori detection

Methods for $H$. pylori detection displayed different power in accuracy, which consequently might affect the detection rate of $H$. pylori infection. Methods for diagnosis of $H$. pylori were classified as invasive tests and noninvasive tests [21]. Invasive tests included rapid urease test, histology, and culture, and the noninvasive tests included ${ }^{13} \mathrm{C}$ or ${ }^{14} \mathrm{C}$ urea breath test, stool antigen detection, and serological approaches for antibodies of $H$. pylori. For the serological tests of anti-H. pylori IgG or/and IgA antibody in serum, high rates of false-positive results may happen and they cannot identify the differences between the current infection and past infection $[21,22]$. So we typically sorted the studies with detection method of serological test into one subgroup and others into the other subgroup as they could identify the current infection precisely.

The studies of current infection group comprised of 51 articles and showed a significant higher prevalence of $H$. pylori infection in DM patients as compared to that in non-DM individuals with $\mathrm{OR}=1.92$ (95\% CI: $1.57-2.34, P<0.001$ ). Similarly, by enrolling 21 articles in serological test group, we found that the infection rate was 53.7\% (1956/3640) in DM group while $46.4 \%(4097 / 8829)$ in the non-DM one $(\mathrm{OR}=1.40,95 \% \mathrm{CI}: 1.10-1.79, P<0.001$; Figure 5$)$. The heterogeneities in both groups were high among studies with $I^{2}=89 \%$ and $I^{2}=81 \%$, respectively (Figure 5).

3.3. Publication Bias. Funnel plot analysis did not show significant evidences of publication bias (Figure 6). Most of the studies were concentrated symmetrically. No significant publication bias was detected by Begg's test with $\operatorname{Pr}=0.411$ 
TABLE 1: Characteristics of the included studies.

\begin{tabular}{|c|c|c|c|c|c|c|c|}
\hline Author & Year & Country & Study design & Type of DM & Age (years) & Method of detection* & NOS \\
\hline Han et al. [20] & 2016 & China & Cross-sectional & T2DM & $64.1 \pm 8.6$ & 1 & 9 \\
\hline Kayar et al. [7] & 2015 & Turkey & Case-control & $\mathrm{T} 2 \mathrm{DM}$ & $18-65$ & 2 & 7 \\
\hline Vafaeimanesh et al. [10] & 2015 & Iran & Cross-sectional & $\mathrm{T} 2 \mathrm{DM}$ & $52.84 \pm 8.82$ & 3 & 7 \\
\hline Zhou et al. [14] & 2015 & China & Case-control & $\mathrm{T} 2 \mathrm{DM}$ & $42.4 \pm 9.8$ & 3,4 & 9 \\
\hline Qiao et al. [45] & 2015 & China & Case-control & $\mathrm{T} 2 \mathrm{DM}$ & $52.5 \pm 1.7$ & 1 & 7 \\
\hline Ji et al. [46] & 2015 & China & Case-control & $\mathrm{T} 2 \mathrm{DM}$ & $51.6 \pm 12.5$ & 1,3 & 8 \\
\hline Bajaj et al. [9] & 2014 & India & Case-control & $\mathrm{T} 2 \mathrm{DM}$ & $\geq 18$ & $3,4,5$ & 8 \\
\hline Chobot et al. [47] & 2014 & Poland & Case-control & T1DM & $13.4 \pm 3.4$ & 1 & 7 \\
\hline Sotuneh et al. [15] & 2014 & Iran & Cross-sectional & $\mathrm{DM}$ & Elderly & 3 & 8 \\
\hline Yang et al. [11] & 2014 & Taiwan & Cross-sectional & $\mathrm{T} 2 \mathrm{DM}$ & $59.6 \pm 10.0$ & 5 & 9 \\
\hline Zhang et al. [48] & 2014 & China & Case-control & $\mathrm{DM}$ & $52.14 \pm 10.25$ & 1 & 7 \\
\hline Wei et al. [49] & 2014 & China & Case-control & $\mathrm{T} 2 \mathrm{DM}$ & $52.79 \pm 12.86$ & 1 & 7 \\
\hline Ye and $\mathrm{Xu}[50]$ & 2014 & China & Case-control & $\mathrm{T} 2 \mathrm{DM}$ & $54.2 \pm 2.0$ & 1 & 7 \\
\hline Liu et al. [51] & 2014 & China & Case-control & $\mathrm{T} 2 \mathrm{DM}$ & $51-65$ & 1 & 7 \\
\hline Zhou et al. [52] & 2014 & China & Case-control & $\mathrm{T} 2 \mathrm{DM}$ & $57.8 \pm 11.7$ & 1 & 7 \\
\hline Wang $\mathrm{F}$ and Wang XF [53] & 2014 & China & Case-control & $\mathrm{T} 2 \mathrm{DM}$ & $54.6 \pm 1.4$ & 1 & 7 \\
\hline Bai et al. [54] & 2014 & China & Case-control & $\mathrm{T} 2 \mathrm{DM}$ & $52.5 \pm 14.2$ & 1 & 7 \\
\hline Jia et al. [55] & 2014 & China & Case-control & $\mathrm{DM}$ & $61.0 \pm 10.0$ & 1 & 6 \\
\hline Jafarzadeh et al. [56] & 2013 & Iran & Cross-sectional & $\mathrm{DM}$ & $42.86 \pm 6.42$ & 3 & 7 \\
\hline Keramat et al. [57] & 2013 & Iran & Case-control & $\mathrm{DM}$ & $51.20 \pm 11.60$ & $3,4,5$ & 8 \\
\hline Xue et al. [58] & 2013 & China & Case-control & T2DM & $57.03 \pm 11.29$ & 1 & 7 \\
\hline Luo H [59] & 2013 & China & Case-control & $\mathrm{DM}$ & $51.5 \pm 4.9$ & 4 & 6 \\
\hline Candelli et al. [60] & 2012 & Italy & Prospective cohort & T1DM & $19.8 \pm 4.3$ & 1 & 7 \\
\hline Jeon et al. [32] & 2012 & USA & Prospective cohort & $\mathrm{DM}$ & $67.9(64.1-71.3)$ & 3 & 7 \\
\hline Oluyemi et al. [61] & 2012 & Nigeria & Cross-sectional & $\mathrm{T} 2 \mathrm{DM}$ & $56.4 \pm 10.4$ & 2 & 7 \\
\hline Hao et al. [62] & 2012 & China & Case-control & $\mathrm{DM}$ & $47.24 \pm 8.49$ & 1 & 6 \\
\hline Xu et al. [63] & 2012 & China & Case-control & $\mathrm{T} 2 \mathrm{DM}$ & $61.0 \pm 10.96$ & 3 & 7 \\
\hline El-Eshmawy et al. [40] & 2011 & Egypt & Case-control & T1DM & $19.35 \pm 2.6$ & 3 & 7 \\
\hline Wan et al. [64] & 2011 & China & Case-control & $\mathrm{T} 2 \mathrm{DM}$ & $53.4 \pm 1.8$ & 1 & 6 \\
\hline Chen et al. [65] & 2011 & China & Case-control & $\mathrm{DM}$ & $53.0 \pm 5.6$ & 1 & 6 \\
\hline Agrawal et al. [66] & 2010 & India & Case-control & $\mathrm{T} 2 \mathrm{DM}$ & - & 5 & 7 \\
\hline Devrajani et al. [8] & 2010 & Pakistan & Case-control & $\mathrm{T} 2 \mathrm{DM}$ & $>35$ & 2 & 7 \\
\hline Ibrahim et al. [44] & 2010 & Egypt & Case-control & $\mathrm{T} 2 \mathrm{DM}$ & $45 \pm 5.4$ & $4,5,6$ & 6 \\
\hline Sfarti et al. [37] & 2010 & Romania & Case-control & T1DM & $49.5 \pm 14.2$ & $1,4,5$ & 7 \\
\hline Xu et al. [67] & 2010 & China & Case-control & $\mathrm{T} 2 \mathrm{DM}$ & $51.5 \pm 13.0$ & 1 & 7 \\
\hline Cabral et al. [68] & 2009 & Brazil & Case-control & T1DM & $17.6 \pm 1.5$ & 5 & 6 \\
\hline Ciortescu et al. [69] & 2009 & Romania & Case-control & DM & - & $1,3,5$ & \# \\
\hline Krause et al. [38] & 2009 & Israel & Case-control & T1DM & $16.0 \pm 8.7$ & 3 & 6 \\
\hline Lazaraki et al. [70] & 2009 & Greece & Case-control & $\mathrm{T} 2 \mathrm{DM}$ & $65.32 \pm 8.56$ & 4,5 & 6 \\
\hline Zhang LQ and Zhang MQ [71] & 2009 & China & Case-control & $\mathrm{T} 2 \mathrm{DM}$ & $56.5 \pm 1.1$ & 1 & 7 \\
\hline Yu $[72]$ & 2009 & China & Case-control & $\mathrm{T} 2 \mathrm{DM}$ & $52.5 \pm 13.4$ & 1 & 6 \\
\hline Ariizumi et al. [73] & 2008 & Japan & Case-control & DM & $62.5 \pm 11.5$ & $3,4,5$ & 6 \\
\hline Demir et al. [74] & 2008 & Turkey & Case-control & $\mathrm{T} 2 \mathrm{DM}$ & $52 \pm 8.2$ & 5 & 6 \\
\hline Hamed et al. [75] & 2008 & Egypt & Case-control & DM & $47.65 \pm 1.2$ & 3 & 7 \\
\hline Nicholas et al. [76] & 2008 & Nigeria & Case-control & $\mathrm{T} 2 \mathrm{DM}$ & $29-72$ & 3 & 7 \\
\hline Yan et al. [77] & 2008 & China & Case-control & $\mathrm{T} 2 \mathrm{DM}$ & $32-85$ & 1 & 6 \\
\hline Wang et al. [78] & 2008 & China & Case-control & $\mathrm{T} 2 \mathrm{DM}$ & $47.1 \pm 6.37$ & 5 & 6 \\
\hline Ji YF et al. [79] & 2008 & China & Case-control & T2DM & $55.2 \pm 13.5$ & 5 & 7 \\
\hline
\end{tabular}


TABLE 1: Continued.

\begin{tabular}{|c|c|c|c|c|c|c|c|}
\hline Author & Year & Country & Study design & Type of DM & Age (years) ${ }^{\star}$ & Method of detection* & NOS \\
\hline Bener et al. [80] & 2007 & Qatar & Case-control & $\mathrm{T} 2 \mathrm{DM}$ & $48.1 \pm 7.9$ & 3 & 7 \\
\hline Sun et al. [81] & 2007 & China & Case-control & $\mathrm{T} 2 \mathrm{DM}$ & $35-85$ & 1 & 7 \\
\hline Jaber [82] & 2006 & Saudi Arabia & Case-control & T1DM & Children & 3 & 7 \\
\hline Lu et al. [83] & 2006 & China & Case-control & T2DM & $59.4 \pm 11.2$ & 3 & 7 \\
\hline Gulcelik et al. [84] & 2005 & Turkey & Case-control & T2DM & $51.9 \pm 10.6$ & 5 & 7 \\
\hline Gillum [85] & 2004 & USA & Cross-sectional & $\mathrm{DM}$ & $40-74$ & 3 & 7 \\
\hline Candelli et al. [27] & 2003 & Italy & Case-control & T2DM & $14.8 \pm 5.6$ & 1 & 6 \\
\hline Anastasios et al. [86] & 2002 & Greece & Cross-sectional & $\mathrm{DM}$ & $61.4 \pm 12.3$ & 5 & 6 \\
\hline Cenerelli et al. [87] & 2002 & Italy & Case-control & T2DM & $55.7 \pm 9.7$ & 1 & 7 \\
\hline Colombo et al. [88] & 2002 & Italy & Case-control & T1DM & Children & 3 & \# \\
\hline De Block et al. [36] & 2002 & Belgium & Case-control & T1DM & $41 \pm 12$ & 3,5 & 7 \\
\hline Maule et al. [89] & 2002 & Italy & Case-control & $\mathrm{T} 2 \mathrm{DM}$ & $46-75$ & 1 & 7 \\
\hline Zelenková et al. [90] & 2002 & Czech & Case-control & $\mathrm{DM}$ & - & 3 & $\#$ \\
\hline Ko et al. [91] & 2001 & China & Case-control & T2DM & $49.9 \pm 12.0$ & 4 & 6 \\
\hline Ivandić et al. [92] & 2001 & Croatia & Case-control & $\mathrm{DM}$ & $23-63$ & 5 & 6 \\
\hline Ravera et al. [93] & 2001 & Uganda & Case-control & DM & - & 5 & 6 \\
\hline Marrollo et al. [94] & 2001 & Italy & Case-control & DM & 63 & 5 & 7 \\
\hline Quatrini et al. [95] & 2001 & Italy & Case-control & $\mathrm{DM}$ & 58 & 1 & 7 \\
\hline Senturk et al. [39] & 2001 & Turkey & Case-control & T2DM & - & 5,6 & $\#$ \\
\hline Vazeou et al. [96] & 2001 & UK & Case-control & T1DM & 14.5 & 3 & 6 \\
\hline Xia [97] & 2001 & Australia & Case-control & $\mathrm{DM}$ & $60.7 \pm 13.3$ & 3 & 7 \\
\hline Zhao [98] & 2001 & China & Case-control & $\mathrm{T} 2 \mathrm{DM}$ & $59.6 \pm 1.3$ & 1 & 6 \\
\hline Arslan et al. [99] & 2000 & Turkey & Case-control & T1DM & Children & 3 & $\#$ \\
\hline Dore et al. [100] & 2000 & Italy & Case-control & $\mathrm{DM}$ & $12-75$ & 3 & 6 \\
\hline Güvener et al. [101] & 1999 & Turkey & Case-control & T2DM & - & 5 & 7 \\
\hline Salardi et al. [102] & 1999 & Italy & Case-control & T1DM & 12 & 3 & 7 \\
\hline de Luis et al. [103] & 1998 & Spain & Case-control & $\mathrm{DM}$ & $24.05 \pm 8.3$ & 3 & 6 \\
\hline Gasbarrini et al. [104] & 1998 & Italy & Case-control & $\mathrm{DM}$ & $35 \pm 11$ & 1 & 7 \\
\hline Gentile et al. [105] & 1998 & Italy & Case-control & T2DM & $51 \pm 8$ & 5 & 7 \\
\hline Pocecco et al. [106] & 1997 & Italy & Case-control & $\mathrm{DM}$ & 16 & 4 & 6 \\
\hline Małlecki et al. [107] & 1996 & Poland & Case-control & $\mathrm{DM}$ & - & 5 & 6 \\
\hline
\end{tabular}

NOS: Newcastle-Ottawa scale. ${ }^{\star}$ Mean age or the range of age in DM group. ${ }^{*} 1={ }^{13} \mathrm{C}$ or ${ }^{14} \mathrm{C}$ urea breath test, $2=$ stool antigen test, $3=$ anti- $H$. pylori antibody, $4=$ rapid urease test, $5=$ histology or biopsy, $6=$ culture. ${ }^{\#}$ Non-English or non-Chinese article or only abstract available which could not get the full text for scoring.

but a significant bias was detected by Egger's test with $P<0.001$ (Figure 7). As Egger's test indicated the possibility of publication bias, the trim and fill method procedure was performed to identify and correct the publication bias. There was 14 hypothetical missing studies indicated by the trim and fill procedure, and the imputed pooled estimate was 1.366 (95\% CI: $1.181-1.580, P<0.001)$. There still existed a statistically significant association between $H$. pylori infection and DM after adjusting for the publication bias, which suggested that our result was credible. Adjusted funnel plot by the trim and fill method was symmetrical and shown in Figure 8 .

\section{Discussion}

DM is a chronic disease characterized by a long-term inflammation mechanism. Guo et al. demonstrated that diabetes was a risk factor for $H$. pylori infection [23]. Several metaanalyses aiming to investigate the association between $H$. pylori infection and DM have been carried out. Zhou et al. recruited 41 studies involving 14,080 patients, and the analysis reported higher risk of $H$. pylori infection among DM patients with $\mathrm{OR}=1.33$ (95\% CI: 1.08-1.64) [12]. Wang et al. retrieved 39 studies involving more than 20,000 participants, with the $\mathrm{OR}=1.59$ (95\% CI: 1.33-1.90) [24]. Our meta-analysis was an updated one and included more studies and individuals. Consistently, we found that the prevalence of $H$. pylori infection was significantly higher in DM patients. But we brought more robust result with higher OR $(\mathrm{OR}=1.69$, 95\% CI: 1.47-1.95; Figure 2). Moreover, we explored more databases and recruited 25 studies reported in Chinese with high-quality score of NOS (all of them were $>5$ ). In addition, in subgroup analysis, we found no significant difference in prevalence of $H$. pylori infection 


\begin{tabular}{|c|c|c|c|c|c|c|c|c|c|c|}
\hline Study or subgroup & \multicolumn{2}{|c|}{ DM } & $\begin{array}{l}\text { Non- } \\
\text { Events }\end{array}$ & $\begin{array}{l}\text {-DM } \\
\text { Total }\end{array}$ & Weight & $\begin{array}{l}\text { Odds ratio } \\
\text { M-H, Random, 95\% CI }\end{array}$ & Year & & $\begin{array}{r}\text { Odds } \\
\text { M-H, Rand }\end{array}$ & $\begin{array}{l}\text { s ratio } \\
\text { dom, } 95 \% \text { CI }\end{array}$ \\
\hline Han et al. 2016 & 3254 & 6395 & 12,041 & 24,415 & $1.7 \%$ & $1.06(1.01,1.12)$ & 2016 & & & 1 \\
\hline Ji et al. 2015 & 83 & 125 & 73 & 142 & $1.4 \%$ & $1.87(1.14,3.07)$ & 2015 & & & \\
\hline Zhou et al. 2015 & 106 & 188 & 28 & 65 & $1.3 \%$ & $1.71(0.97,3.02)$ & 2015 & & & \\
\hline Vafaeimanesh et al. 2015 & 139 & 211 & 110 & 218 & $1.5 \%$ & $1.90(1.28,2.80)$ & 2015 & & & \\
\hline Qiao et al. 2015 & 25 & 42 & 9 & 20 & $0.9 \%$ & $1.80(0.61,5.27)$ & 2015 & & & \\
\hline Kayar et al. 2015 & 40 & 62 & 31 & 71 & $1.2 \%$ & $2.36(1.16,4.73)$ & 2015 & & & \\
\hline Bai et al. 2014 & 102 & 150 & 80 & 150 & $1.4 \%$ & $1.86(1.16,2.97)$ & 2014 & & & \\
\hline Yang et al. 2014 & 147 & 238 & 358 & 729 & $1.6 \%$ & $1.67(1.24,2.26)$ & 2014 & & & {[} \\
\hline Chobot et al. 2014 & 17 & 149 & 49 & 298 & $1.3 \%$ & $0.65(0.36 .1 .18)$ & 2014 & & & - \\
\hline Jia et al. 2014 & 50 & 100 & 15 & 37 & $1.1 \%$ & $1.47(0.68,3.15)$ & 2014 & & & \\
\hline Wang F and Wang XF 2014 & 52 & 80 & 40 & 80 & $1.3 \%$ & $1.86(0.98,3.50)$ & 2014 & & & \\
\hline Bajaj et al. 2014 & 62 & 80 & 35 & 60 & $1.2 \%$ & $2.46(1.18,5.13)$ & 2014 & & & \\
\hline Liu et al. 2014 & 240 & 281 & 41 & 86 & $1.4 \%$ & $6.42(3.75,11.00)$ & 2014 & & & \\
\hline Zhou et al. 2014 & 148 & 200 & 71 & 180 & $1.5 \%$ & $4.37(2.836 .75)$ & 2014 & & & \\
\hline Sotuneh et al. 2014 & 303 & 391 & 688 & 909 & $1.6 \%$ & $1.11(0.83,1.47)$ & 2014 & & & 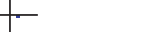 \\
\hline Wei et al. 2014 & 68 & 109 & 51 & 106 & $1.4 \%$ & $1.79(1.04,3.08)$ & 2014 & & & \\
\hline Ye and Xu 2014 & 84 & 110 & 54 & 120 & $1.3 \%$ & $3.95(2.24,6.97)$ & 2014 & & & \\
\hline Zhang et al. 2014 & 168 & 300 & 62 & 200 & $1.5 \%$ & $2.83(1.94,4.13)$ & 2014 & & & \\
\hline Keramat et al. 2013 & 58 & 79 & 53 & 84 & $1.2 \%$ & $1.62(0.83,3.15)$ & 2013 & & & \\
\hline Xue et al. 2013 & 79 & 120 & 60 & 120 & $1.4 \%$ & $1.93(1.15,3.24)$ & 2013 & & & \\
\hline Jafarzadeh et al. 2013 & 76 & 100 & 76 & 100 & $1.3 \%$ & $1.06(0.55,2.01)$ & 2013 & & & \\
\hline Luo 2013 & 36 & 49 & 34 & 62 & $1.1 \%$ & $2.28(1.02,5.11)$ & 2013 & & & \\
\hline Candelli et al. 2012 & 17 & 69 & 7 & 99 & $1.0 \%$ & $4.30(1.67,11.04)$ & 2012 & & & \\
\hline Oluyemi et al. 2012 & 18 & 100 & 13 & 100 & $1.1 \%$ & $1.47(0.68,3.19)$ & 2012 & & & \\
\hline Hao et al. 2012 & 145 & 227 & 227 & 436 & $1.6 \%$ & $1.63(1.17,2.26)$ & 2012 & & & \\
\hline Jeon et al. 2012 & 139 & 144 & 580 & 638 & $1.0 \%$ & $2.78(1.09,7.06)$ & 2012 & & & \\
\hline $\mathrm{Xu}$ et al. 2012 & 58 & 130 & 18 & 50 & $1.2 \%$ & $1.43(0.73,2.81)$ & 2012 & & & \\
\hline El-Eshmawy et al. 2011 & 128 & 162 & 41 & 80 & $1.3 \%$ & $3.58(2.01,6.39)$ & 2011 & & & \\
\hline Chen et al. 2011 & 51 & 62 & 43 & 74 & $1.1 \%$ & $3.34(1.50,7.43)$ & 2011 & & & \\
\hline Wan et al. 2011 & 92 & 120 & 59 & 130 & $1.4 \%$ & $3.95(2.29,6.83)$ & 2011 & & & \\
\hline Xu et al. 2010 & 430 & 768 & 65 & 172 & $1.6 \%$ & $2.09(1.49,2.94)$ & 2010 & & & \\
\hline Agrawal et al. 2010 & 50 & 80 & 32 & 80 & $1.3 \%$ & $2.50(1.32,4.72)$ & 2010 & & & \\
\hline Devrajani et al. 2010 & 54 & 74 & 38 & 74 & $1.2 \%$ & $2.56(1.29,5.08)$ & 2010 & & & \\
\hline Ibrahim et al. 2010 & 53 & 98 & 58 & 102 & $1.4 \%$ & $0.89(0.64,3.35)$ & 2010 & & & \\
\hline Sfarti et al. 2010 & 49 & 69 & 25 & 40 & $1.1 \%$ & $1.47(0.64,3.35)$ & 2010 & & & \\
\hline Lazaraki et al. 2009 & 20 & 49 & 12 & 29 & $1.0 \%$ & $0.98(0.38,2.48)$ & 2009 & & & \\
\hline Zhang LQ Zhang MQ 2009 & 100 & 160 & 76 & 160 & $1.5 \%$ & $1.84(1.18,2.88)$ & 2009 & & & \\
\hline Ciortescu et al. 2009 & 70 & 100 & 73 & 100 & $1.3 \%$ & $0.86(0.47,1.60)$ & 2009 & & & \\
\hline Cabral et al. 2009 & 5 & 15 & 17 & 30 & $07 \%$ & $0.38(0.10,1.39)$ & 2009 & & & \\
\hline Krause et al. 2009 & 31 & 57 & 113 & 140 & $1.2 \%$ & $0.28(0.15,0.56)$ & 2009 & & & \\
\hline Yu 2009 & 135 & 180 & 80 & 150 & $1.4 \%$ & $2.63(1.65,4.18)$ & 2009 & & & \\
\hline Demir et al. 2008 & 87 & 141 & 83 & 142 & $1.4 \%$ & $1.15(0.71,1.84)$ & 2008 & & & \\
\hline Hamed et al. 2008 & 68 & 80 & 46 & 60 & $1.1 \%$ & $1.72(0.73,4.06)$ & 2008 & & & \\
\hline Ariizumi et al. 2008 & 36 & 67 & 46 & 67 & $1.2 \%$ & $0.53(0.26,1.07)$ & 2008 & & & \\
\hline Yan et al. 2008 & 113 & 150 & 36 & 70 & $1.3 \%$ & $2.88(1.59,5.24)$ & 2008 & & & \\
\hline Wang et al. 2008 & 65 & 103 & 72 & 175 & $1.4 \%$ & $2.45(1.48,4.04)$ & 2008 & & & \\
\hline Ji YF et al. 2008 & 81 & 120 & 76 & 110 & $1.4 \%$ & $0.93(0.53,1.62)$ & 2008 & & & \\
\hline Nicholas et al. 2008 & 21 & 60 & 17 & 60 & $1.1 \%$ & $1.36(0.63,2.95)$ & 2008 & & & \\
\hline Sun et al. 2007 & 76 & 230 & 54 & 150 & $1.5 \%$ & $0.88(0.57,1.35)$ & 2007 & & & \\
\hline Bener et al. 2007 & 161 & 210 & 5 & 210 & $1.5 \%$ & $1.79(1.17,2.74)$ & 2007 & & & \\
\hline Lu et al. 2006 & 74 & 132 & 136 & 24 & $0.9 \%$ & $4.85(1.71,13.76)$ & 2006 & & & \\
\hline Jaber 2006 & 21 & 61 & 128 & 543 & $1.3 \%$ & $1.70(0.97,2.99)$ & 2006 & & & \\
\hline Gulcelik et al. 2005 & 59 & 78 & 33 & 71 & 1.25 & $3.58(1.78,7.17)$ & 2005 & & & \\
\hline Gillum 2004 & 193 & 366 & 1628 & 4218 & $1.6 \%$ & $1.77(1.43,2.20)$ & 2004 & & & \\
\hline Candelli et al. 2003 & 34 & 121 & 43 & 147 & $1.4 \%$ & $0.95(0.56,1.61)$ & 2003 & & & \\
\hline Cenerelli et al. 2002 & 13 & 30 & 18 & 43 & $1.0 \%$ & $1.06(0.41,2.73)$ & 2002 & & & \\
\hline Colombo et al. 2002 & 41 & 138 & 45 & 138 & $1.4 \%$ & $0.87(0.52,1.45)$ & 2002 & & & \\
\hline De Block et al. 2002 & 72 & 229 & 42 & 100 & $1.4 \%$ & $0.63(0.39,1.03)$ & 2002 & & & \\
\hline Zelenková et al. 2002 & 53 & 195 & 110 & 216 & $1.5 \%$ & $0.36(0.24,0.54)$ & 2002 & & & \\
\hline Maule et al. 2002 & 22 & 31 & 15 & 31 & $0.9 \%$ & $2.61(0.91,7.43)$ & 2002 & & & \\
\hline Anastasios et al. 2002 & 25 & 67 & 37 & 105 & $1.3 \%$ & $1.09(0.58,2.07)$ & 2002 & & & \\
\hline Ravera et al. 2001 & 2 & 22 & 8 & 110 & $0.6 \%$ & $0.16(0.03,0.70)$ & 2001 & & & \\
\hline Vazeou et al. 2001 & 8 & 118 & 43 & 171 & $0.9 \%$ & $1.48(0.54,4.07)$ & 2001 & & & \\
\hline Ko et al. 2001 & 32 & 63 & 31 & 55 & $1.2 \%$ & $0.80(0.39,1.65)$ & 2001 & & & \\
\hline Zhao 2001 & 230 & 370 & 19 & 255 & $1.4 \%$ & $20.41(12.22,34.07)$ & 2001 & & & \\
\hline Xia et al. 2001 & 142 & 429 & 54 & 170 & $1.5 \%$ & $1.06(0.73,1.55)$ & 2001 & & & \\
\hline Marrollo et al. 2001 & 48 & 74 & 56 & 117 & $1.3 \%$ & $2.01(1.10,3.66)$ & 2001 & & & \\
\hline Quantrini et al. 2001 & 49 & 71 & 33 & 71 & $1.2 \%$ & $2.56(1.26,5.09)$ & 2001 & & & \\
\hline Ivabdić et al. 2001 & 31 & 46 & 8 & 40 & $0.9 \%$ & $8.27(3.07,22.25)$ & 2001 & & & \\
\hline Senturk et al. 2001 & 59 & 67 & 58 & 72 & $1.0 \%$ & $1.78(0.69,4.56)$ & 2001 & & & \\
\hline Arslan et al. 2000 & 49 & 88 & 13 & 42 & $1.1 \%$ & $2.80(1.29,6.10)$ & 2000 & & & \\
\hline Dore et al. 2000 & 195 & 385 & 223 & 506 & $1.6 \%$ & $1.30(1.00,1.70)$ & 2000 & & & \\
\hline Guvener et al. 1999 & 41 & 51 & 14 & 25 & $0.9 \%$ & $3.22(1.13,9.20)$ & 1999 & & & \\
\hline Salardi et al. 1999 & 18 & 103 & 25 & 236 & $1.3 \%$ & $1.79(0.93,3.44)$ & 1999 & & & \\
\hline Gentile et al. 1998 & 122 & 164 & 82 & 164 & $1.4 \%$ & $2.90(1.82,4.63)$ & 1998 & & & \\
\hline de Luis et al. 1998 & 38 & 80 & 34 & 100 & $1.3 \%$ & $1.76(0.96,3.21)$ & 1998 & & & \\
\hline Gasbarrini et al. 1998 & 43 & 116 & 17 & 50 & $1.2 \%$ & $1.14(0.57,2.29)$ & 1998 & & & \\
\hline Pocecco et al. 1997 & 18 & 69 & 17 & 310 & $1.2 \%$ & $6.08(2.94,12.58)$ & 1997 & & & \\
\hline Mallecki et al. 1996 & 12 & 39 & 68 & 100 & $1.1 \%$ & $0.21(0.09,0.47)$ & 1996 & & & \\
\hline Total (95\% CI) & & 17,187 & & 40,210 & $100.0 \%$ & $1.69[1.47,1.95]$ & & & & \\
\hline Total events & 9434 & & 19108 & & & & & & & \\
\hline Heterogeneity: $\tau^{2}=0.30 ; \chi^{2}$ & $2=574$ & $28, \mathrm{df}=$ & $=78(P<$ & 0.0000 & $1) ; I^{2}=8($ & & & 0.1 & 0.2 & 5 \\
\hline Test for overall effect: $Z=7$. & $7.29(P$ & $<0.0000$ & & & & & & & $\mathrm{DM}$ & Contro \\
\hline
\end{tabular}

FIGURE 2: Forest plot for pooled prevalence of $H$. pylori infection in DM group and non-DM group. 


\begin{tabular}{|c|c|c|c|c|c|c|c|c|c|c|c|}
\hline \multirow[b]{2}{*}{ Study or subgroup } & \multicolumn{2}{|c|}{$\mathrm{DM}$} & \multicolumn{2}{|c|}{ Non-DM } & \multicolumn{3}{|c|}{ Odds ratio } & \multirow{2}{*}{\multicolumn{4}{|c|}{$\begin{array}{c}\text { Odds ratio } \\
\text { M-H, Random, 95\% CI }\end{array}$}} \\
\hline & Events & Total & Events & Total & Weight & M-H, Random, 95\% CI & Year & & & & \\
\hline \multicolumn{12}{|l|}{$\mathrm{T} 2 \mathrm{DM}$} \\
\hline Han et al. 2016 & 3254 & 6395 & 12,041 & 24,415 & $3.0 \%$ & $1.06(1.01,1.12)$ & 2016 & & & $=$ & \\
\hline Ji et al. 2015 & 83 & 125 & 73 & 142 & $2.6 \%$ & $1.87(1.14,3.07)$ & 2015 & & & & \\
\hline Vafaeimanesh et al. 2015 & 139 & 211 & 110 & 218 & $2.7 \%$ & $1.90(1.28,2.80)$ & 2015 & & & & \\
\hline Qiao et al. 2015 & 25 & 42 & 9 & 20 & $1.7 \%$ & $1.80(0.61,5.27)$ & 2015 & & & & \\
\hline Zhou et al. 2015 & 106 & 188 & 28 & 65 & $2.5 \%$ & $1.71(0.97,3.02)$ & 2015 & & & & \\
\hline Bai et al. 2014 & 102 & 150 & 80 & 150 & $2.6 \%$ & $1.86(1.16,2.97)$ & 2014 & & & & \\
\hline Yang et al. 2014 & 147 & 238 & 358 & 729 & $2.9 \%$ & $1.67(1.24,2.26)$ & 2014 & & & & \\
\hline Bajaj et al. 2014 & 62 & 80 & 35 & 60 & $2.2 \%$ & $2.46(1.18,5.13)$ & 2014 & & & & \\
\hline Wang $F$ and Wang XF 2014 & 52 & 80 & 40 & 60 & $2.4 \%$ & $1.86(0.98,3.50)$ & 2014 & & & & \\
\hline Zhou et al. 2014 & 148 & 200 & 71 & 180 & $2.7 \%$ & $4.37(2.83,6.75)$ & 2014 & & & & \\
\hline Liu et al. 2014 & 240 & 281 & 41 & 86 & $2.5 \%$ & $6.42(3.75,11.00)$ & 2014 & & & & $\rightarrow$ \\
\hline Wei et al. 2014 & 68 & 109 & 51 & 106 & $2.5 \%$ & $1.79(1.04,3.08)$ & 2014 & & & & \\
\hline Ye and Xu 2014 & 84 & 110 & 51 & 120 & $2.5 \%$ & $3.95(2.24,6.97)$ & 2014 & & & & \\
\hline Xu et al. 2012 & 58 & 130 & 18 & 50 & $2.3 \%$ & $1.43(0.73,2.81)$ & 2012 & & & & \\
\hline Oluyemi et al. 2012 & 18 & 100 & 13 & 100 & $2.1 \%$ & $1.47(0.68,3.19)$ & 2012 & & & & \\
\hline Wan et al. 2011 & 92 & 120 & 59 & 130 & $2.5 \%$ & $3.95(2.29,6.83)$ & 2011 & & & & \\
\hline Agrawal et al. 2010 & 50 & 80 & 32 & 80 & $2.4 \%$ & $2.50(1.32,4.72)$ & 2010 & & & & \\
\hline Xu et al. 2010 & 430 & 768 & 65 & 172 & $2.8 \%$ & $2.09(1.49,2.94)$ & 2010 & & & & \\
\hline Ibrahim et al. 2010 & 53 & 98 & 58 & 102 & $2.5 \%$ & $0.89(0.51,1.56)$ & 2010 & & & & \\
\hline Devrajani et al. 2010 & 54 & 74 & 38 & 74 & $2.3 \%$ & $2.56(1.29,5.08)$ & 2010 & & & & \\
\hline Yu 2009 & 135 & 180 & 80 & 150 & $2.6 \%$ & $2.63(1.65,4.18)$ & 2009 & & & & \\
\hline Zhang LQ and Zhang MQ 2009 & 9100 & 160 & 76 & 160 & $2.7 \%$ & $1.84(1.18,2.88)$ & 2009 & & & & \\
\hline Lazaraki et al. 2009 & 20 & 49 & 12 & 29 & $1.9 \%$ & $0.98(0.38,2.48)$ & 2009 & & & & \\
\hline Wang et al. 2008 & 65 & 103 & 72 & 175 & $2.6 \%$ & $2.45(1.48,4.04)$ & 2008 & & & & \\
\hline Yan et al. 2008 & 113 & 150 & 36 & 70 & $2.4 \%$ & $2.88(1.59,5.24)$ & 2008 & & & & \\
\hline Nicholas et al. 2008 & 21 & 60 & 17 & 60 & $2.1 \%$ & $1.36(0.63,2.95)$ & 2008 & & & & \\
\hline Ji YF et al. 2008 & 81 & 120 & 76 & 110 & $2.5 \%$ & $0.93(0.53,1.62)$ & 2008 & & & & \\
\hline Demir et al. 2008 & 87 & 141 & 83 & 142 & $2.6 \%$ & $1.15(0.71,1.84)$ & 2008 & & & & \\
\hline Bener et al. 2007 & 161 & 210 & 136 & 210 & $2.7 \%$ & $1.79(1.17,2.74)$ & 2007 & & & & \\
\hline Gulcelik et al. 2005 & 59 & 78 & 33 & 71 & $2.3 \%$ & $3.58(1.78,7.17)$ & 2005 & & & & \\
\hline Candelli et al. 2003 & 34 & 121 & 43 & 147 & $2.5 \%$ & $0.95(0.56,1.61)$ & 2003 & & & & \\
\hline Maule et al. 2002 & 22 & 31 & 15 & 31 & $1.7 \%$ & $2.61(0.91,7.43)$ & 2002 & & & & \\
\hline Cenerelli et al. 2002 & 13 & 30 & 18 & 43 & $1.8 \%$ & $1.06(0.41,2.73)$ & 2002 & & & & \\
\hline Zhao 2001 & 230 & 370 & 19 & 255 & $2.6 \%$ & $20.41(12.22,34.07)$ & 2001 & & & & $>$ \\
\hline Ko et al. 2001 & 32 & 63 & 31 & 55 & $2.2 \%$ & $0.80(0.39,1.65)$ & 2001 & & & & \\
\hline Senturk et al. 2001 & 59 & 67 & 58 & 72 & $1.9 \%$ & $1.78(0.69,4.56)$ & 2001 & & & & \\
\hline Güvener et al. 1999 & 41 & 51 & 14 & 25 & $1.7 \%$ & $3.2(1.13,9.20)$ & 1999 & & & & \\
\hline Gentile et al. 1998 & 122 & 164 & 82 & 164 & $2.6 \%$ & $2.90(1.82,4.63)$ & 1998 & & & & \\
\hline Total (95\% CI) & & 12,271 & & 29,413 & $100.0 \%$ & $2.05(1.67,2.52)$ & & & & & \\
\hline Total events & 6929 & & 14,325 & & & & & & & & \\
\hline \multicolumn{12}{|c|}{ Heterogeneity: $\tau^{2}=0.36 ; \chi^{2}=361.48, \mathrm{df}=41(P<0.00001) ; I^{2}=89 \%$} \\
\hline \multicolumn{12}{|c|}{ Test for overall effect: $Z=6.89(P<0.00001)$} \\
\hline \multicolumn{12}{|c|}{ T1DM } \\
\hline Chobot et al. 2014 & 17 & 149 & 49 & 298 & $9.0 \%$ & $0.65(0.36,1.18)$ & 2014 & & & & \\
\hline Candelli et al. 2012 & 17 & 69 & 7 & 99 & $7.4 \%$ & $4.30(1.67,11.04)$ & 2012 & & & & $\rightarrow$ \\
\hline EI-Eshmawy et al. 2011 & 128 & 162 & 41 & 80 & $9.1 \%$ & $3.58(2.01,6.39)$ & 2011 & & & & \\
\hline Sfarti et al. 2010 & 49 & 69 & 25 & 40 & $7.9 \%$ & $1.47(0.64,3.35)$ & 2010 & & & & \\
\hline Krause et al. 2009 & 31 & 57 & 113 & 140 & $8.7 \%$ & $0.28(0.15,0.56)$ & 2009 & & & & \\
\hline Cabral et al. 2009 & 5 & 15 & 17 & 30 & $5.8 \%$ & $0.38(0.10,1.39)$ & 2009 & & & & \\
\hline Jaber 2006 & 21 & 61 & 128 & 543 & $9.2 \%$ & $1.70(0.97,2.99)$ & 2006 & & & & \\
\hline De Block et al. 2002 & 72 & 299 & 42 & 100 & $9.5 \%$ & $0.63(0.39,1.03)$ & 2002 & & & & \\
\hline Colombo et al. 2002 & 41 & 138 & 45 & 138 & $9.4 \%$ & $1.87(0.52,1.45)$ & 2002 & & & & \\
\hline Vazeou et al. 2001 & 8 & 118 & 8 & 171 & $7.1 \%$ & $1.48(0.54,4.07)$ & 2001 & & & & \\
\hline Arslan et al. 2000 & 49 & 88 & 13 & 42 & $8.2 \%$ & $2.80(1.29,6.10)$ & 2000 & & & & \\
\hline Salardi et al. 1999 & 18 & 103 & 25 & 236 & $8.7 \%$ & $1.79(0.93,3.44)$ & 1999 & & & & \\
\hline Total $(95 \% \mathrm{CI})$ & & 1258 & & 1917 & $100.0 \%$ & $1.23[0.77,1.96]$ & & & & & \\
\hline Total events & 456 & & 513 & & & & & & & & \\
\hline \multicolumn{8}{|c|}{ Heterogeneity: $\tau^{2}=0.53 ; \chi^{2}=61.39, \mathrm{df}=11(P<0.00001) ; I^{2}=82 \%$} & 0.1 & $\begin{array}{ll}1 & 1 \\
0.2 & 0.5\end{array}$ & 25 & 10 \\
\hline Test for overall effect: $Z=0.87(P$ & $=0.38)$ & & & & & & & & DM & Control & \\
\hline
\end{tabular}

FIGURE 3: Forest plot for subgroup analysis based on types of DM. 


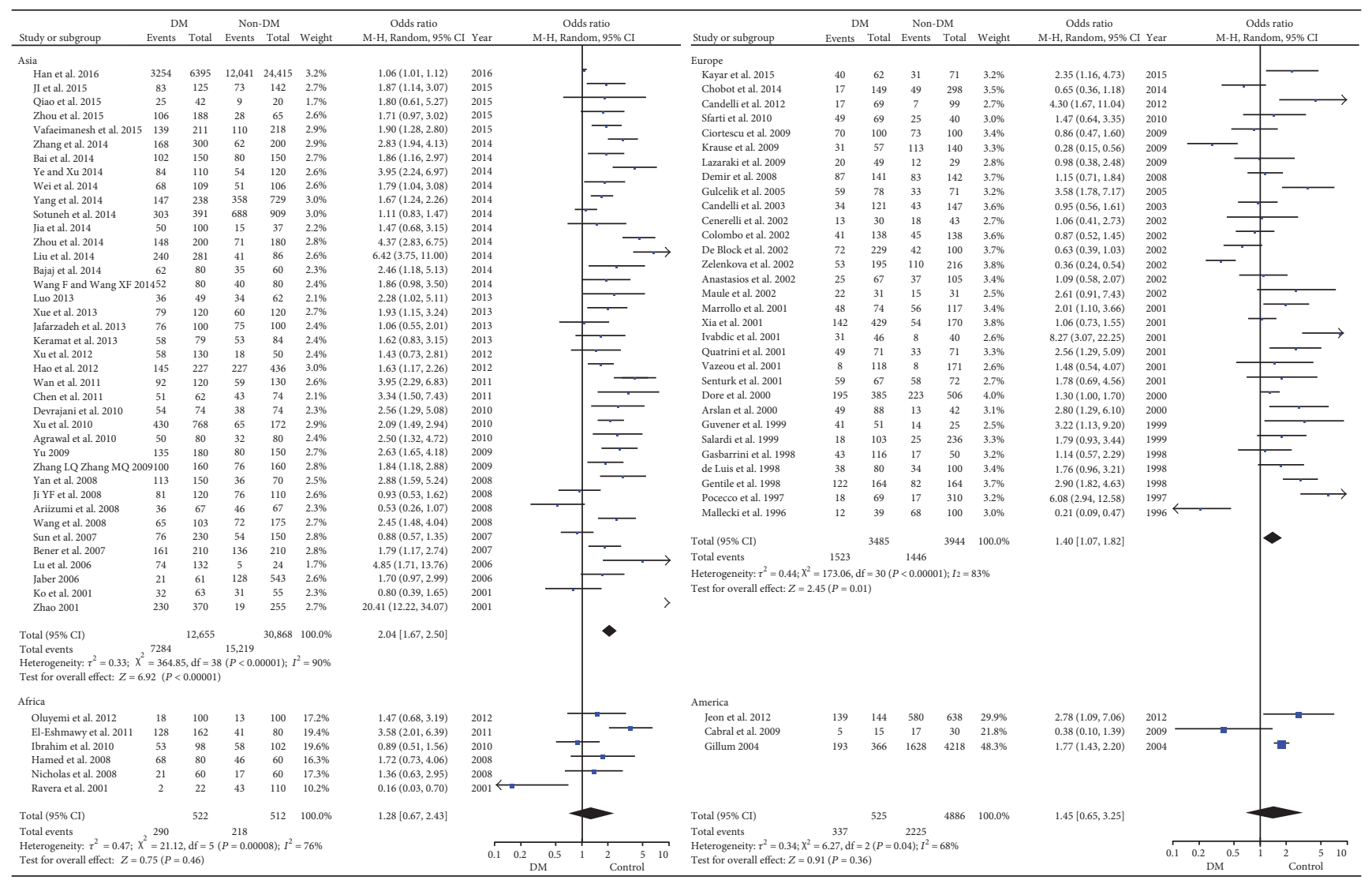

FIGURE 4: Forest plot for subgroup analysis based on geographic regions. (India, Japan, China, Qatar, Pakistan, Saudi Arabia Iran, Hong Kong, and Taiwan were included in group Asia. Greece, Turkey, Italy, Poland, Romania, Belgium, Spain, Croatia, Israel, UK, and Czech Republic were included in group Europe, as well as Australia because it comprises similar races and people who lived in similar lifestyle with these countries. Brazil and USA were included in group America. Egypt and Nigeria were included in group Africa.)

in comparison between T1DM patients and non-DM people, which was inconsistent with what was reported by Wang et al. In a subgroup analysis of geographical regions, we found significant higher $H$. pylori infection rate among DM individuals in group Asia and group Europe but not in group Africa or group America. It was inconsistent with the Zhou et al. study which reported that the H. pylori effect only happened in Asian people. In this meta-analysis, we found no publication bias with Begg's test, while Egger's test showed a possibility of publication bias. But we performed the trim and fill method and found 14 hypothetical missing studies. The imputed pooled result still supported our original one. Therefore, no publication bias was shown in our meta-analysis and the result we got was credible. In this meta-analysis, the study of Han et al., even though with a total of 30,810 participants, did not affect the significance of the pooled results. Maybe it was because the other studies recruited as enough individuals (a total of 26,587 participants) as to be commensurate to the scale of the Han et al. study. Furthermore, the quality score of NOS for the study Han et al. was 9, which was high. Hence, despite the overweight scale, the study of Han et al. should not be neglected.

We found that there existed an association between H. pylori infection and DM in this meta-analysis. Several possible mechanisms might explain the association.
Hyperglycemic condition in diabetic individuals could result in immune dysfunction, including damage to the neutrophil function, depression of antioxidant system, and impaired humoral immunity [25]. Moreover, abnormal enteric neuropathy caused by high blood sugar can modulate immune-cell function and stimulate proinflammatory cytokine production, resulting in neurodegeneration [26]. It leads to delay gastric emptying and lacking of acid secretion, which promotes bacterial colonization or overgrowth in gastrointestinal tract [27]. On the other hand, H. pylori infection in diabetic patients may worsen glycemic control [28], which leads to the difficulty of DM treatment, forming the vicious circle.

In this meta-analysis, we found that DM patients had a higher prevalence of $H$. pylori infection. But we could not come to the result whether and what role $H$. pylori infection plays on the pathogenesis or development of DM. It was reported that patients could be coinfected with $H$. pylori and some other pathogens like herpes simplex virus 1, cytomegalovirus, and Epstein-Barr virus, some of whom were also associated with DM [29-31]. But the number of researches on this issue was limited. We could not know whether other pathogens affect the effect of $H$. pylori on DM, either. Jeon et al. firstly carried out a prospective cohort study of 782 Latino elderly aged $>60$ years and 


\begin{tabular}{|c|c|c|c|c|c|c|c|c|c|c|c|}
\hline \multirow{3}{*}{$\begin{array}{l}\text { Study or subgroup } \\
\text { Current infection group }\end{array}$} & \multicolumn{2}{|c|}{ DM } & Non-1 & $\mathrm{DM}$ & & Odds ratio & & & O & ratio & \\
\hline & Events & Total & Events & Total & Weight & $\mathrm{M}-\mathrm{H}$, Random, $95 \% \mathrm{CI}$ & Year & & M-H, Ra & om, $95 \% \mathrm{CI}$ & \\
\hline & & & & & & & & & & & \\
\hline Han et al. 2016 & 3254 & 6395 & 12,041 & 24,415 & $2.5 \%$ & $1.06(1.01,1.12)$ & 2016 & & & $=$ & \\
\hline Qiao et al. 2015 & 25 & 42 & 9 & 20 & $1.5 \%$ & $1.80(0.61,5.27)$ & 2015 & & & & \\
\hline Kayar et al. 2015 & 40 & 62 & 31 & 71 & $1.9 \%$ & $2.35(1.16,4.73)$ & 2015 & & & & \\
\hline Liu et al. 2014 & 240 & 281 & 41 & 86 & $2.1 \%$ & $6.42(3.75,11.00)$ & 2014 & & & & $\rightarrow$ \\
\hline Zhou et al. 2014 & 148 & 200 & 71 & 180 & $2.3 \%$ & $4.37(2.83,6.75)$ & 2014 & & & & \\
\hline Bai et al. 2014 & 102 & 150 & 80 & 150 & $2.2 \%$ & $1.86(1.16,2.97)$ & 2014 & & & & \\
\hline Yang et al. 2014 & 147 & 238 & 358 & 729 & $2.4 \%$ & $1.67(1.24,2.26)$ & 2014 & & & & \\
\hline Chobot et al. 2014 & 17 & 149 & 49 & 298 & $2.1 \%$ & $0.65(0.36,1.18)$ & 2014 & & & & \\
\hline Jia et al. 2014 & 50 & 100 & 15 & 37 & $1.8 \%$ & $1.47(0.68,3.15)$ & 2014 & & & & \\
\hline Wang et al. 2014 & 52 & 80 & 40 & 80 & $2.0 \%$ & $1.86(0.98,3.50)$ & 2014 & & & & \\
\hline Zhang et al. 2014 & 168 & 300 & 62 & 200 & $2.3 \%$ & $2.83(1.94,4.13)$ & 2014 & & & & \\
\hline Ye and Xu. 2014 & 84 & 110 & 54 & 120 & $2.1 \%$ & $3.95(2.24,6.97)$ & 2014 & & & & \\
\hline Wei et al. 2014 & 68 & 109 & 51 & 106 & $2.1 \%$ & $1.79(1.04,3.08)$ & 2014 & & & & \\
\hline Xue et al. 2013 & 79 & 120 & 60 & 120 & $2.2 \%$ & $1.93(1.15,3.24)$ & 2013 & & & & \\
\hline Luo 2013 & 36 & 49 & 34 & 62 & $1.8 \%$ & $2.28(1.02,5.11)$ & 2013 & & & & \\
\hline Hao et al. 2012 & 145 & 227 & 227 & 436 & $2.4 \%$ & $1.63(1.17,2.26)$ & 2012 & & & & \\
\hline Oluyemi et al. 2012 & 18 & 100 & 13 & 100 & $1.8 \%$ & $1.47(0.68,3.19)$ & 2012 & & & & \\
\hline Candelli et al. 2012 & 17 & 69 & 7 & 99 & $1.6 \%$ & $4.30(1.67,11.04)$ & 2012 & & & & \\
\hline Wan et al. 2011 & 92 & 120 & 59 & 130 & $2.1 \%$ & $3.95(2.29,6.83)$ & 2011 & & & & \\
\hline Chen et al. 2011 & 51 & 62 & 43 & 74 & $1.8 \%$ & $3.34([1.50,7.43)$ & 2011 & & & & \\
\hline Sfarti et al. 2010 & 49 & 69 & 25 & 40 & $1.8 \%$ & $1.47(0.64,3.35)$ & 2010 & & & & \\
\hline Ibrahim et al. 2010 & 53 & 98 & 58 & 102 & $2.1 \%$ & $0.89(0.51,1.56)$ & 2010 & & & & \\
\hline Xu 2010 & 430 & 768 & 65 & 172 & $2.4 \%$ & $2.09(1.49,2.94)$ & 2010 & & & & \\
\hline Devrajani et al. 2010 & 54 & 74 & 38 & 74 & $1.9 \%$ & $2.56(1.29,5.08)$ & 2010 & & & & \\
\hline Agrawal et al. 2010 & 50 & 80 & 32 & 80 & $2.0 \%$ & $2.50(1.32,4.72)$ & 2010 & & & & \\
\hline Cabral et al. 2009 & 5 & 15 & 17 & 30 & $1.2 \%$ & $0.38(0.10,1.39)$ & 2009 & & & & \\
\hline Yu 2009 & 135 & 180 & 80 & 150 & $2.2 \%$ & $2.63(1.65,4.18)$ & 2009 & & & & \\
\hline Zhang LQ and Zhang MQ 2009 & 100 & 160 & 76 & 160 & $2.2 \%$ & $1.84(1.18,2.88)$ & 2009 & & & & \\
\hline Lazaraki et al. 2009 & 20 & 49 & 12 & 29 & $1.6 \%$ & $0.98(0.38,2.48)$ & 2009 & & & & \\
\hline Demir et al. 2008 & 87 & 141 & 83 & 142 & $2.2 \%$ & $1.15(0.71,1.84)$ & 2008 & & & & \\
\hline Yan et al. 2008 & 113 & 150 & 36 & 70 & $2.1 \%$ & $2.88(1.59,5.24)$ & 2008 & & & & \\
\hline Wang et al. 2008 & 65 & 103 & 72 & 175 & $2.2 \%$ & $2.45(1.48,4.04)$ & 2008 & & & & \\
\hline Ji YF et al. 2008 & 81 & 120 & 76 & 110 & $2.1 \%$ & $0.93(0.53,1.62)$ & 2008 & & & 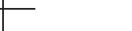 & \\
\hline Sun et al. 2007 & 76 & 230 & 54 & 150 & $2.3 \%$ & $0.88(0.57,1.35)$ & 2007 & & & & \\
\hline Gulcelik et al. 2005 & 59 & 78 & 33 & 71 & $1.9 \%$ & $3.58(1.78,7.17)$ & 2005 & & & & \\
\hline Candelli et al. 2003 & 34 & 121 & 43 & 147 & $2.1 \%$ & $0.95(0.56,1.61)$ & 2003 & & & & \\
\hline Cenerelli et al. 2002 & 13 & 30 & 18 & 43 & $1.6 \%$ & $1.06(0.41,2.73)$ & 2002 & & & & \\
\hline Maule et al. 2002 & 22 & 31 & 15 & 31 & $1.5 \%$ & $2.61(0.91,7.43)$ & 2002 & & & & \\
\hline Anastasios et al. 2002 & 25 & 67 & 37 & 105 & $2.0 \%$ & $1.09(0.58,2.07)$ & 2002 & & & & \\
\hline Zhao 2001 & 230 & 370 & 19 & 255 & $2.2 \%$ & $20.41(12.22,34.07)$ & 2001 & & & & $>$ \\
\hline Ravera et al. 2001 & 2 & 22 & 43 & 110 & $1.0 \%$ & $0.16(0.03,0.70)$ & 2001 & & & & \\
\hline Ko et al. 2001 & 32 & 63 & 31 & 55 & $1.9 \%$ & $0.80(0.39,1.65)$ & 2001 & & & & \\
\hline Marrollo et al. 2001 & 48 & 74 & 56 & 117 & $2.1 \%$ & $2.01(1.10,3.66)$ & 2001 & & & & \\
\hline Senturk et al. 2001 & 59 & 67 & 58 & 72 & $1.6 \%$ & $1.78(0.69,4.56)$ & 2001 & & & & \\
\hline Quatrini et al. 2001 & 49 & 71 & 33 & 71 & $1.9 \%$ & $2.56(1.29,5.09)$ & 2001 & & & & \\
\hline Ivabdic et al. 2001 & 31 & 46 & 8 & 40 & $1.6 \%$ & $8.27(3.07,22.25)$ & 2001 & & & & \\
\hline Guvener et al. 1999 & 41 & 51 & 14 & 25 & $1.5 \%$ & $3.22(1.13,9.20)$ & 1999 & & & & \\
\hline Gasbarrini et al. 1998 & 43 & 116 & 17 & 50 & $1.9 \%$ & $1.14(0.57,2.29)$ & 1998 & & & & \\
\hline Gentile et al. 1998 & 122 & 164 & 82 & 164 & $2.2 \%$ & $2.90(1.82,4.63)$ & 1998 & & & & \\
\hline Pocecco et al. 1997 & 18 & 69 & 17 & 310 & $1.9 \%$ & $6.08(2.94,12.58)$ & 1997 & & & & $\rightarrow$ \\
\hline Mallecki et al. 1996 & 12 & 39 & 68 & 100 & $1.8 \%$ & $0.21(0.09,0.47)$ & 1996 & & & & \\
\hline Total $(95 \% \mathrm{CI})$ & & 12,679 & & 30,763 & $100.0 \%$ & $1.92[1.57,2.34]$ & & & & 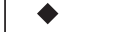 & \\
\hline Total events & 6991 & & 14,661 & & & & & & & & \\
\hline Heterogeneity: $\tau^{2}=0.40 ; \chi^{2}=448$ & $56, \mathrm{df}=$ & $0(P<0$ & 001); $I^{2}=$ & $=89 \%$ & & & & & & & \\
\hline Test for overall effect: $Z=6.44(P$ & $<0.0000$ & & & & & & & & & & \\
\hline Serological test group & & & & & & & & & & & \\
\hline Vafaeimanesh et al. 2015 & 139 & 211 & 110 & 218 & $5.7 \%$ & $1.90(1.28,2.80)$ & 2015 & & & & \\
\hline Sotuneh et al. 2014 & 303 & 391 & 688 & 909 & $6.1 \%$ & $1.11(0.83,1.47)$ & 2014 & & & & \\
\hline Jafarzadeh et al. 2013 & 76 & 100 & 75 & 100 & $4.6 \%$ & $1.06(0.55,2.01)$ & 2013 & & & & \\
\hline Xu et al. 2012 & 58 & 130 & 18 & 50 & $4.4 \%$ & $1.43(0.73,2.81)$ & 2012 & & & & \\
\hline Jeon et al. 2012 & 139 & 144 & 580 & 638 & $3.4 \%$ & $2.78(1.09,7.06)$ & 2012 & & & & \\
\hline EI-Eshmawy et al. 2011 & 128 & 162 & 41 & 80 & $4.9 \%$ & $3.58(2.01,6.39)$ & 2011 & & & & \\
\hline Krause et al. 2009 & 31 & 57 & 113 & 140 & $4.4 \%$ & $0.28(0.15,0.56)$ & 2009 & & & & \\
\hline Hamed et al. 2008 & 68 & 80 & 46 & 60 & $3.7 \%$ & $1.72(0.73,4.06)$ & 2008 & & & & \\
\hline Nicholas et al. 2008 & 21 & 60 & 17 & 60 & $4.0 \%$ & $1.36(0.36,2.95)$ & 2008 & & & & \\
\hline Bener et al. 2007 & 161 & 210 & 136 & 210 & $5.5 \%$ & $1.79(1.17,2.74)$ & 2007 & & & & \\
\hline Jaber 2006 & 21 & 61 & 128 & 543 & $4.9 \%$ & $1.70(0.97,2.99)$ & 2006 & & & & \\
\hline Lu et al. 2006 & 74 & 132 & 5 & 24 & $3.0 \%$ & $4.85(1.71,13.76)$ & 2006 & & & & \\
\hline Gillum et al. 2004 & 193 & 366 & 1628 & 4218 & $6.3 \%$ & $1.77(1.43,2.20)$ & 2004 & & & - & \\
\hline Colombo et al. 2002 & 41 & 138 & 45 & 138 & $5.2 \%$ & $0.87(0.52,1.45)$ & 2002 & & & & \\
\hline Zelenkova et al. 2002 & 53 & 195 & 110 & 216 & $5.6 \%$ & $0.36(0.24,0.54)$ & 2002 & & & & \\
\hline Vazeou et al. 2001 & 8 & 118 & 8 & 171 & $3.1 \%$ & $1.48(0.54,4.07)$ & 2001 & & & & \\
\hline Xia et al. 2001 & 142 & 429 & 54 & 170 & $5.7 \%$ & $1.06(0.73,1.55)$ & 2001 & & & - & \\
\hline Arslan et al. 2000 & 49 & 88 & 13 & 42 & $4.0 \%$ & $2.80(1.29,6.10)$ & 2000 & & & 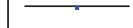 & \\
\hline Dore et al. 2000 & 195 & 385 & 223 & 506 & $6.2 \%$ & $1.30(1.00,1.70)$ & 2000 & & & - & \\
\hline Salardi et al. 1999 & 18 & 103 & 25 & 236 & $4.5 \%$ & $1.79(0.93,3.44)$ & 1999 & & & & \\
\hline de Luis et al. 1998 & 38 & 80 & 34 & 100 & $4.7 \%$ & $1.76(0.96,3.21)$ & 1998 & & & & \\
\hline Total $(95 \% \mathrm{CI})$ & & 3640 & & 8829 & $100.0 \%$ & $1.40[1.10,1.79]$ & & & & $>$ & \\
\hline Total events & 1956 & & 4097 & & & & & & & & \\
\hline $\begin{array}{l}\text { Heterogeneity: } \tau^{2}=0.23 ; \chi^{2}=102 \\
\text { Test for overall effect: } Z=2.71(P\end{array}$ & $\begin{array}{l}67, \mathrm{df}= \\
=0.007)\end{array}$ & $0(P<0$ & $001) ; I^{2}=$ & $=81 \%$ & & & & & $0.2 \quad 0.5$ & 2 & 10 \\
\hline
\end{tabular}

FIGURE 5: Forest plot for subgroup analysis of methods for $H$. pylori detection. 


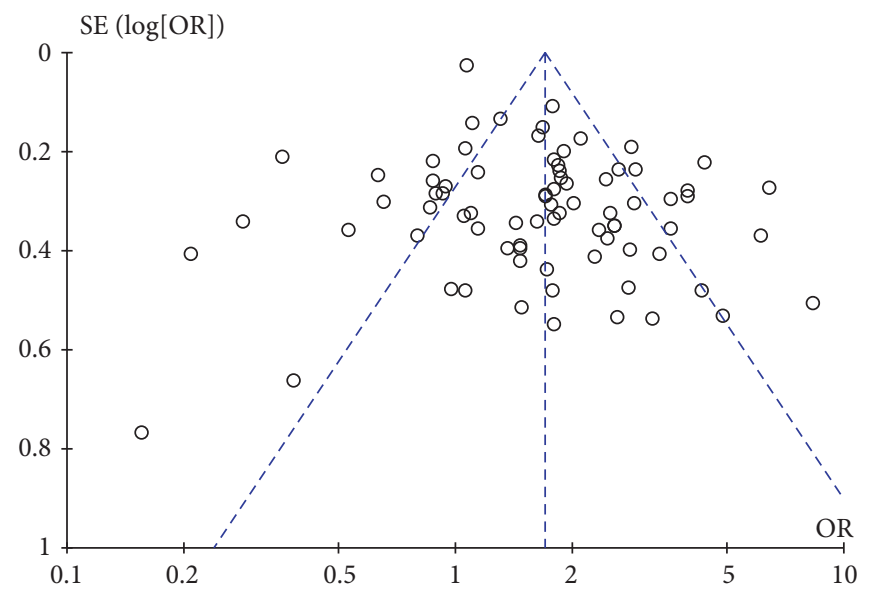

Figure 6: Funnel plot of this meta-analysis.
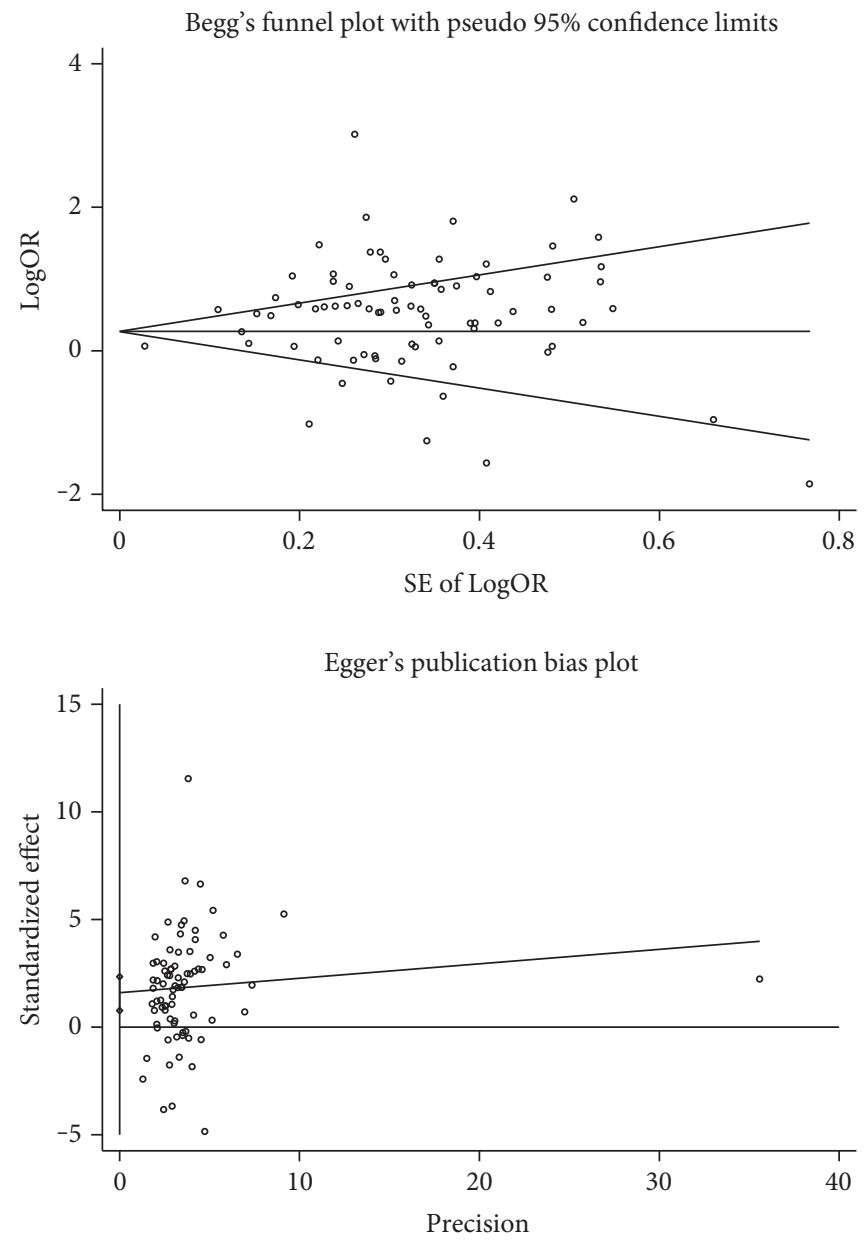

Figure 7: Begg's and Egger's funnel plot of this meta-analysis.

diabetes-free [32]. After following up over 10 years, the authors demonstrated that $H$. pylori seropositive patients experienced a greater rate of incident DM than individuals without DM (hazard ratio 2.69, 95\% CI: 1.10-6.60), whereas those who were seropositive for herpes simplex virus 1 , varicella virus, cytomegalovirus, and Toxoplasma. gondii did not show an increased rate of DM. It indicated that $H$. pylori infection might play an unknown role in the pathogenesis of DM, which implicated a potential step for preventing DM by eradication of $H$. pylori infection. Moreover, it also suggested that other pathogens such as cytomegalovirus and herpes simplex virus 1 might not 


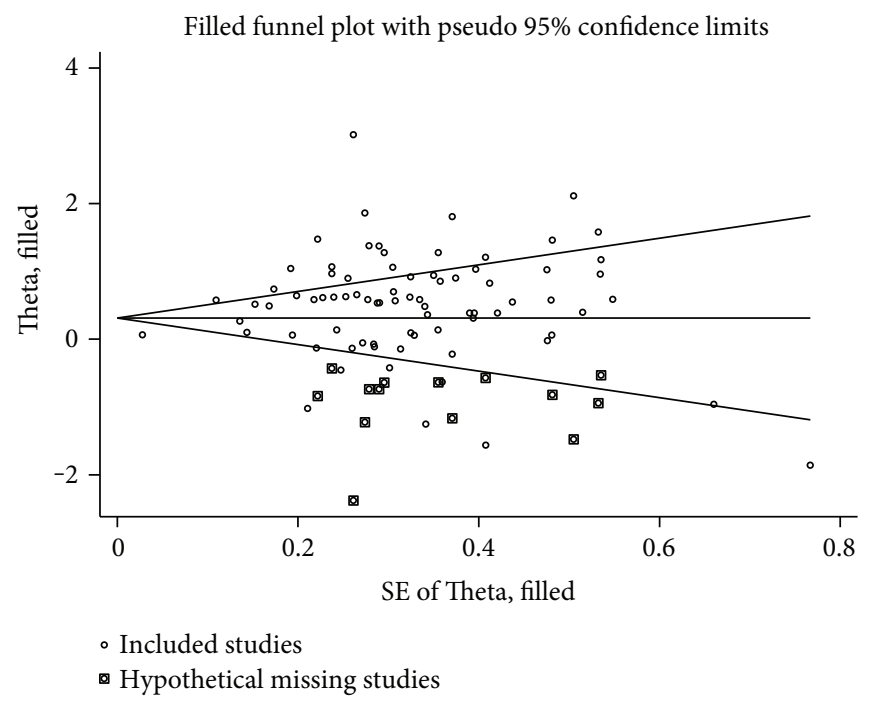

FIGURE 8: Adjusted funnel plot in the trim and fill method of this meta-analysis.

have the similar effect on the DM like H. pylori. But our meta-analysis just revealed the association between $H$. pylori and DM, but could not suggest the effect of $H$. pylori on DM pathogenesis. More researches are needed to find out the actually effect of $H$. pylori infection on DM.

In subgroup analysis based on the types of DM, we demonstrated that $56.5 \%$ T2DM individuals were infected with $H$. pylori, but only $36.2 \%$ T1DM carried the bacterium (Figure 3). T2DM was more significantly prone to the infection of $H$. pylori. As to T2DM, insulin resistant (IR) is one of its characteristics. Aydemir et al. showed that IR was significantly higher in $H$. pylori infection group [33]. And Eshraghian et al. also supported that $H$. pylori infection was a risk factor for IR [34, 35]. Furthermore, it was reported that IR in T2DM patients could be improved after successful eradication of $H$. pylori [4]. It might partly explain the higher $H$. pylori infection rate in T2DM patients. On the other hand, we found no significant difference in prevalence of $H$. pylori infection in comparison between T1DM patients and non-DM people $(P=0.38)$, consistently with the report by Candelli et al. [27]. Whether this outcome is caused by the different pathogenesis or the onset age of T1DM and T2DM remains unclear. In the T1DM group, the mean age in most studies was not over 20, except for the studies of De Block et al. [36] and Sfarti et al. [37], while in T2DM group, the mean age was usually over 50 years old (Table 1). Epidemiological studies suggested that the prevalence of $H$. pylori infection increases with age [34]. As T1DM mainly onsets during childhood or young age, T1DM patients probably have less chance to be exposed to $H$. pylori infection. Consistently, Krause et al. showed a significantly lower positive rate of antibodies against $H$. pylori in T1DM patients [38]. But some studies held the contrary view that T1DM individuals were also prone to $H$. pylori infection [39, 40]. However, our meta-analysis with pooled estimate favored that T2DM rather than T1DM was associated with $H$. pylori infection. But the sample size of T1DM subgroup was not as large as that of T2DM. Larger sample size is needed to further verify the association between $H$. pylori infection and DM, especially T1DM.

The prevalence of $H$. pylori infection varies in different regions. We found significant higher $H$. pylori infection rate among DM individuals in group Asia and group Europe but not in group Africa or group America (Figure 4). Firstly, it was to be noted that there were much bigger sample size in group Asia and group Europe, respectively. This might be due to the more accurate detection methods and in group Africa and group America; the sample size might be too small to draw robust conclusion. Secondly, it might be explained by that the condition of medical care in developing countries from group Asia was too poor for DM patients to get good control of DM and prevent infectious complications. On the other hand, the epidemiology and different strains of $H$. pylori infection might attribute to the part of the result. Epidemiology studies revealed that almost all the Asians are infected with the strain of $H$. pylori carrying cytotoxinassociated gene A (CagA) but only nearly $60 \%$ of western people carried this stain $[41,42]$. It was reported that $H$. pylori infection in Asians was predominated by CagA iceA1 genotypes while Americans and Africans by $\mathrm{CagA}$ iceA2 genotypes [41, 43]. CagA is a major virulence factor of $H$. pylori and has been reported to be associated with diabetic complications [44]. CagA-positive strain of $H$. pylori could cause poor glycemic control in T2DM and difficulty in eradication, which might result in the visible $H$. pylori effect among Asian but not African DM patients. However, due to the lack of data, we could not carry out the subgroup analysis based on different strains of $H$. pylori.

A number of testing methods are available for $H$. pylori detection. Serological test, namely, anti-H. pylori IgG and/ or IgA test, is not affected by acid suppression therapy or recent antibiotic use. But seropositivity could not confirm current $H$. pylori infection, and anti-H. pylori IgG titre usually remains elevated for a long period even after clearance or eradication. Some study using anti-H. pylori IgG as the diagnosis of $H$. pylori infection might overestimate 
the infection rate. We typically conducted the analysis of serological test group and current infection group and found that in both subgroups, DM patients had higher prevalence of $H$. pylori infection than non-DM people (Figure 5). As a result, the association between $H$. pylori infection and DM was verified despite of different methods for $H$. pylori detection.

Despite the robust result, there existed limitations in our study. The studies were highly heterogeneous. Variables like age, sex, race, economic status, DM prevalence, and strains of $H$. pylori infection in the included studies varied. For the lack of enough detailed data, subgroup analysis stratified by age, sex, different stages of DM, and strains of H. pylori, which might bring up heterogeneity, could not be carried out. Furthermore, most of the articles meeting the inclusive criteria were case-control or cross-sectional ones, and only 3 were prospective ones. More well-designed and prospective cohort studies are needed for clarifying the association between $H$. pylori infection and DM.

In conclusion, despite the limitations, our meta-analysis suggested that there is significantly higher prevalence of H. pylori infection in DM when compared with the non-DM individuals. And the difference is associated with type $2 \mathrm{DM}$ but not type $1 \mathrm{DM}$.

\section{Abbreviations}

$\begin{array}{ll}\text { H. pylori: } & \text { Helicobacter pylori } \\ \text { DM: } & \text { Diabetes mellitus } \\ \text { T2DM: } & \text { Type 2 DM } \\ \text { T1DM: } & \text { Type 1 DM } \\ \text { NOS: } & \text { Newcastle-Ottawa scale } \\ \text { OR: } & \text { Odds ratio } \\ \text { CI: } & \text { Confidence interval } \\ \text { RR: } & \text { Risk ratio } \\ \text { IR: } & \text { Insulin resistant } \\ \text { CagA: } & \text { Cytotoxin-associated gene A } \\ \text { MALT: } & \text { Mucosa-associated lymphoid tissue. }\end{array}$

\section{Conflicts of Interest}

The authors declare that they have no conflict of interest.

\section{Authors' Contributions}

Jun-Zhen Li and Jie-Yao Li contributed equally to this work.

\section{Acknowledgments}

This study was supported by the National Natural Science Foundation of China (no. 81270442 and no. 81370475).

\section{References}

[1] World Gastroenterology Organisation, "World Gastroenterology Organisation Global Guideline: Helicobacter pylori in developing countries," Journal of Clinical Gastroenterology, vol. 45, no. 5, pp. 383-388, 2011.

[2] F. Franceschi, G. Zuccalà, D. Roccarina, and A. Gasbarrini, "Clinical effects of Helicobacter pylori outside the stomach,"
Nature Reviews Gastroenterology \& Hepatology, vol. 11, no. 4, pp. 234-242, 2014.

[3] F. Franceschi, A. Gasbarrini, S. A. Polyzos, and J. Kountouras, "Extragastric diseases and Helicobacter pylori," Helicobacter, vol. 20, Supplement 1, pp. 40-46, 2015.

[4] L. R. Malamug, R. Karnchanasorn, R. Samoa, and K. C. Chiu, "The role of Helicobacter pylori seropositivity in insulin sensitivity, beta cell function, and abnormal glucose tolerance," Scientifica (Cairo), vol. 2014, Article ID 870165, 7 pages, 2014.

[5] A. Kondrashova and H. Hyöty, "Role of viruses and other microbes in the pathogenesis of type 1 diabetes," International Reviews of Immunology, vol. 33, no. 4, pp. 284-295, 2014.

[6] L. Simon, J. Tornóczky, M. Tóth, M. Jámbor, and Z. Sudár, "The significance of Campylobacter pylori infection in gastroenterologic and diabetic practice," Orvosi Hetilap, vol. 130, no. 25, pp. 1325-1329, 1989.

[7] Y. Kayar, Ö. Pamukçu, H. Eroğlu, K. Kalkan Erol, A. Ilhan, and O. Kocaman, "Relationship between Helicobacter pylori infections in diabetic patients and inflammations, metabolic syndrome, and complications," International Journal of Chronic Diseases, vol. 2015, Article ID 290128, 6 pages, 2015.

[8] B. R. Devrajani, S. Z. Shah, A. A. Soomro, and T. Devrajani, "Type 2 diabetes mellitus: a risk factor for Helicobacter pylori infection: ahospital based case-control study," International Journal of Diabetes in Developing Countries, vol. 30, no. 1, pp. 22-26, 2010.

[9] S. Bajaj, L. Rekwal, S. P. Misra, V. Misra, R. K. Yadav, and A. Srivastava, "Association of helicobacter pylori infection with type 2 diabetes," Indian Journal of Endocrinology and Metabolism, vol. 18, no. 5, pp. 694-699, 2014.

[10] J. Vafaeimanesh, M. Parham, and M. Bagherzadeh, "Helicobacter pylori infection prevalence: is it different in diabetics and nondiabetics?" Indian Journal of Endocrinology and Metabolism, vol. 19, no. 3, pp. 364-368, 2015.

[11] G. H. Yang, J. S. Wu, Y. C. Yang, Y. H. Huang, F. H. Lu, and C. J. Chang, "Gastric Helicobacter pylori infection associated with risk of diabetes mellitus, but not prediabetes," Journal of Gastroenterology and Hepatology, vol. 29, no. 10, pp. 1794-1799, 2014.

[12] X. Zhou, C. Zhang, J. Wu, and G. Zhang, "Association between Helicobacter pylori infection and diabetes mellitus a meta-analysis of observational studies," Diabetes Research and Clinical Practice, vol. 99, no. 2, pp. 200-208, 2013.

[13] T. Tamura, E. Morita, S. Kawai et al., "No association between Helicobacter pylori infection and diabetes mellitus among a general Japanese population: a cross-sectional study," SpringerPlus, vol. 4, p. 602, 2015.

[14] F. Zhou, X. Zhong, J. Chen et al., "Helicobacter pylori infection associated with type 2 diabetic nephropathy in patients with dyspeptic symptoms," Diabetes Research and Clinical Practice, vol. 110, no. 3, pp. 328-334, 2015.

[15] N. Sotuneh, S. R. Hosseini, J. Shokri-Shirvani, A. Bijani, and R. Ghadimi, "Helicobacter pylori infection and metabolic parameters: is there an association in elderly population?" International Journal of Preventive Medicine, vol. 5, no. 12, pp. 1537-1542, 2014.

[16] M. K. Swartz, "The PRISMA statement: a guideline for systematic reviews and meta-analyses," Journal of Pediatric Health Care, vol. 25, no. 1, pp. 1-2, 2011. 
[17] G. A. Wells, B. Shea, D. O'Connell et al., The Newcastle-Ottawa Scale (NOS) for Assessing the Quality of Nonrandomised Studies in Meta-Analyses, Department of Epidemiology and Commuunity Medicine, University of Ottawa, http://www.ohri.ca/ programs/clinical_epidemiology/oxford.Asp.

[18] R. SimonianDer and N. Laird, "Meta-analysis in clinical trials," Controlled Clinical Trials, vol. 7, no. 3, pp. 177-188, 1986.

[19] S. Duval and R. Tweedie, "Trim and fill: a simple funnel-plotbased method of testing and adjusting for publication bias in meta-analysis," Biometrics, vol. 56, no. 2, pp. 455-463, 2000.

[20] X. Han, Y. Li, J. Wang et al., "Helicobacter pylori infection is associated with type 2 diabetes among a middle- and oldage Chinese population," Diabetes/Metabolism Research and Reviews, vol. 32, no. 1, pp. 95-101, 2016.

[21] A. Mentis, P. Lehours, and F. Mégraud, "Epidemiology and diagnosis of Helicobacter pylori infection," Helicobacter, vol. 20, Supplement 1, pp. 1-7, 2015.

[22] B. Braden, "Diagnosis of Helicobacter pylori infection," BMJ, vol. 344, article e828, 2012.

[23] X. Guo, B. H. Zhao, and M. X. Zhang, "Risk factors of Helicobacter pylori infection among adults in northern China," Hepato-Gastroenterology, vol. 58, no. 106, pp. 306-310, 2011.

[24] F. Wang, J. Liu, and Z. Lv, “Association of Helicobacter pylori infection with diabetes mellitus and diabetic nephropathy: a meta-analysis of 39 studies involving more than 20,000 participants," Scandinavian Journal of Infectious Diseases, vol. 45, no. 12, pp. 930-938, 2013.

[25] A. Y. Peleg, T. Weerarathna, J. S. McCarthy, and T. M. Davis, "Common infections in diabetes: pathogenesis, management and relationship to glycaemic control," Diabetes/Metabolism Research and Reviews, vol. 23, no. 1, pp. 3-13, 2007.

[26] C. T. Brown, A. G. Davis-Richardson, A. Giongo et al., "Gut microbiome metagenomics analysis suggests a functional model for the development of autoimmunity for type 1 diabetes," PloS One, vol. 6, article e25792, 2011.

[27] M. Candelli, D. Rigante, G. Marietti et al., "Helicobacter pylori, gastrointestinal symptoms, and metabolic control in young type 1 diabetes mellitus patients," Pediatrics, vol. 111, 4, Part 1, pp. 800-803, 2003.

[28] C. Horikawa, S. Kodama, K. Fujihara et al., "Association of Helicobacter pylori infection with glycemic control in patients with diabetes: a meta-analysis," Journal of Diabetes Research, vol. 2014, Article ID 250620, 7 pages, 2014.

[29] L. Baccaglini, V. J. Schoenbach, C. Poole et al., “Association between herpes simplex virus type 1 and Helicobacter pylori in US adolescents," Oral Surgery, Oral Medicine, Oral Pathology, Oral Radiology, and Endodontics, vol. 101, no. 1, pp. 63-69, 2006.

[30] J. Zhang, Y. Y. Liu, H. L. Sun et al., "High human cytomegalovirus IgG level is associated with increased incidence of diabetic atherosclerosis in type 2 diabetes mellitus patients," Medical Science Monitor, vol. 21, pp. 4102-4110, 2015.

[31] S. Singh and H. C. Jha, "Status of Epstein-Barr virus coinfection with Helicobacter pylori in gastric cancer," Journal of Oncology, vol. 2017, Article ID 3456264, 17 pages, 2017.

[32] C. Y. Jeon, M. N. Haan, C. Cheng et al., "Helicobacter pylori infection is associated with an increased rate of diabetes," Diabetes Care, vol. 35, no. 3, pp. 520-525, 2012.

[33] S. Aydemir, T. Bayraktaroglu, M. Sert et al., "The effect of Helicobacter pylori on insulin resistance," Digestive Diseases and Sciences, vol. 50, no. 11, pp. 2090-2093, 2005.
[34] A. Eshraghian, S. A. Hashemi, A. Hamidian Jahromi et al., "Helicobacter pylori infection as a risk factor for insulin resistance," Digestive Diseases and Sciences, vol. 54, no. 9, pp. 1966-1970, 2009.

[35] R. E. Pounder and D. Ng, "The prevalence of Helicobacter pylori infection in different countries," Alimentary Pharmacology \& Therapeutics, vol. 9, Supplement 2, pp. 33-39, 1995.

[36] C. E. BlockDe, I. H. LeeuwDe, J. J. Bogers et al., "Helicobacter pylori, parietal cell antibodies and autoimmune gastropathy in type 1 diabetes mellitus," Alimentary Pharmacology \& Therapeutics, vol. 16, no. 2, pp. 281-289, 2002.

[37] C. Sfarti, A. Trifan, C. Hutanasu, C. Cojocariu, A. M. Singeap, and C. Stanciu, "Prevalence of gastroparesis in type 1 diabetes mellitus and its relationship to dyspeptic symptoms," Journal of Gastrointestinal and Liver Diseases, vol. 19, no. 3, pp. 279284, 2010.

[38] I. Krause, J. M. Anaya, A. Fraser et al., “Anti-infectious antibodies and autoimmune-associated autoantibodies in patients with type I diabetes mellitus and their close family members," Annals of the New York Academy of Sciences, vol. 1173, pp. 633-639, 2009.

[39] O. Senturk, Z. Canturk, B. Cetinarslan, C. Ercin, S. Hulagu, and N. Z. Canturk, "Prevalence and comparisons of five different diagnostic methods for Helicobacter pylori in diabetic patients," Endocrine Research, vol. 27, no. 1-2, pp. 179-189, 2001.

[40] M. M. El-Eshmawy, A. K. El-Hawary, S. S. Abdel Gawad, and A. A. El-Baiomy, "Helicobacter pylori infection might be responsible for the interconnection between type 1 diabetes and autoimmune thyroiditis," Diabetology and Metabolic Syndrome, vol. 3, no. 1, p. 28, 2011.

[41] Y. Yamaoka, T. Kodama, O. Gutierrez, J. G. Kim, K. Kashima, and D. Y. Graham, "Relationship between Helicobacter pylori ice A, cagA, and vacA status and clinical outcome: studies in four different countries," Journal of Clinical Microbiology, vol. 37, no. 7, pp. 2274-2279, 1999.

[42] T. Mizushima, T. Sugiyama, Y. Komatsu, J. Ishizuka, M. Kato, and M. Asaka, "Clinical relevance of the babA2 genotype of Helicobacter pylori in Japanese clinical isolates," Journal of Clinical Microbiology, vol. 39, no. 7, pp. 2463-2465, 2001.

[43] M. Kidd, R. M. Peek, A. J. Lastovica, D. A. Israel, A. F. Kummer, and J. A. Louw, "Analysis of iceA genotypes in South African Helicobacter pylori strains and relationship to clinically significant disease," Gut, vol. 49, no. 5, pp. 629-635, 2001.

[44] A. Ibrahim, T. Zaher, T. A. Ghonemy, S. A. El-Azim, M. A. El-Azim, and A. Ramadan, "Impact of cytotoxin-associated gene A of Helicobacter pylori strains on microalbuminuria in type 2 diabetes," Saudi Journal of Kidney Diseases and Transplantation, vol. 21, no. 4, pp. 694-700, 2010.

[45] Y. Q. Qiao, Q. Wang, and J. X. Liu, "Helicobacter pylori infection in type 2 diabetes: 42 cases (Chinese)," Health Weekly, vol. 1, pp. 10-11, 2015.

[46] S. W. Ji, H. Qu, C. L. Liu, Y. G. Zhang, and J. B. Wang, "Prevalence of Helicobacter pylori infection in 125 patients with type 2 diabetes and its effects on diabetic gastroparesis," Chinese Journal of Digestion, vol. 6, pp. 377-381, 2015.

[47] A. Chobot, K. Bak-Drabik, E. Skała-Zamorowska, A. Krzywicka, J. Kwiecień, and J. Polańska, "Helicobacter pylori infection in type 1 diabetes children and adolescents using ${ }^{13} \mathrm{C}$ urea breath test," Polish Journal of Microbiology, vol. 63, no. 1, pp. 63-67, 2014. 
[48] G. H. Zhang, Q. H. Yi, and J. Ma, "Helicobacter pylori infection in diabetes mellitus: 300 cases (Chinese)," Yunnan Medicine, vol. 3, pp. 388-390, 2014.

[49] Y. N. Wei, H. L. Hei, W. Liu, X. Yang, L. J. Wang, and Y. Su, "The association between Helicobacter pylori infection and type 2 diabetes in the Han population in Henan province, China (Chinese)," Journal of Medical Forum, vol. 2, pp. 9899, 2014.

[50] L. Ye and G. Xu, "Helicobacter pylori infection in diabetic patients (Chinese)," Diabetes New World, vol. 10, p. 27, 2014.

[51] J. X. Liu, X. Q. Zhang, G. Huang et al., "Study on the relationship between the infection of helicobacter pylori and carotid atherosclerosis in patients with type 2 diabetes," Chinese Journal of Difficult and Complicated Cases, vol. 7, pp. 704-706, 2014.

[52] W. H. Zhou, X. D. Mai, H. F. Lu, Y. H. Chen, K. W. Guo, and $\mathrm{X}$. Long, "The correlation studies and clinical analysis of type 2 diabetes and Helicobacter pylori infection," Journal of Taishan Medical College, vol. 8, pp. 738-740, 2014.

[53] F. Wang and X. F. Wang, "The association between Helicobacter pylori infection and diabetes mellitus (Chinese)," Medical Information, vol. 5, 2014.

[54] B. L. Bai, X. T. You, and P. Cao, "The correlation between Helicobacter pylori infection and diabetic peripheral neuropathy in type 2 diabetes (Chinese)," China Health Care \& nutrition, vol. 24, no. 3, 2014.

[55] A. H. Jia, Y. X. Liu, Y. Y. Li et al., "The analysis of necessity of Helicobacter pylori detection in type 2 diabetes (Chinese)," Practical Journal of Medicine \& Pharmacy, vol. 31, no. 8, 2014.

[56] A. Jafarzadeh, M. T. Rezayati, and M. Nemati, "Helicobacter pylori seropositivity in patients with type 2 diabetes mellitus in south-east of Iran," Acta Medica Iranica, vol. 51, no. 12, pp. 892-896, 2013.

[57] F. Keramat, S. H. Hashemi, A. Majlesi, S. Haddadinejad, A. M. Esfehani, and J. Poorolajal, "The association between diabetes mellitus and Helicobacter pylori infection," International Journal of Diabetes in Developing Countries, vol. 33, no. 3, pp. 155-160, 2013.

[58] C. J. Xue, Y. Chen, D. He, W. G. Zhong, and Y. Xia, “The association between Helicobacter pylori infection and type 2 diabetes (Chinese)," Continuing Medical Education, vol. 27, no. 10, pp. 27-28, 2013.

[59] H. Luo, "49 cases on the analyses of Helicobacter pylori infection in diabetes with peptic ulcer (Chinese)," Shanxi Medical Journal, vol. 42, no. 5, 2013.

[60] M. Candelli, D. Rigante, A. Schiavino et al., "High reinfection rate of Helicobacter pylori in young type 1 diabetic patients a three-year follow-up study," European Review for Medical and Pharmacological Sciences, vol. 16, no. 11, pp. 14681472, 2012.

[61] A. Oluyemi, E. Anomneze, S. Smith, and O. Fasanmade, "Prevalence of a marker of active helicobacter pylori infection among patients with type 2 diabetes mellitus in Lagos, Nigeria," BMC Research Notes, vol. 5, p. 284, 2012.

[62] Z. H. Hao, C. Y. Chen, J. M. Wang, H. Q. Chen, and Y. Li, "Helicobacter Pylori infection in patients with diabetes mellitus and the related factors of carotid atherosclerosis," Chinese General Practice, vol. 15, no. 7, pp. 1914-1916, 2012.

[63] Y. B. Xu, X. E. Yan, T. Yi, and M. F. Li, “The correlation between Helicobacter Pylori infection and diabetic retinopathy in type 2 diabetes (Chinese)," Medical Innovation of China, vol. 26, pp. 11-12, 2012.

[64] Y. Wan, F. F. Zhang, and Q. Dong, "Infection of Helicobacter Pylori in type 2 diabetes (Chinese)," Clinical Journal of Medical Officers, vol. 39, no. 6, p. 1054, 2011.

[65] S. Y. Chen, H. Lu, and S. Gao, "Diabetes mellitus with peptic ulcer: Clinic analysis of 62 cases," Practical Journal of Clinical Medicine, vol. 08, no. 5, pp. 122-124, 2011.

[66] R. P. Agrawal, R. Sharma, D. Garg, R. Pokharna, D. K. Kochar, and R. P. Kothari, "Role of Helicobacter pylori in causation of diabetic gastropathies and non-gastrointestinal complications in type 2 diabetes," Journal of the Indian Medical Association, vol. 108, no. 3, pp. 140-143, 2010.

[67] Y. G. Xu, X. Y. Liu, X. Y. Zhu, L. Guo, and S. Z. Wang, "Analysis of Helicobacter pylori infection status in type 2 diabetes mellitus," Chinese Journal of Postgraduates of Medicine, vol. 33, no. 19, 2010.

[68] V. L. Cabral, F. R. Patrício, M. A. Gabbay, S. A. Dib, and S. J. Miszputen, "Intraepithelial lymphocytes in duodenum from Brazilian adolescents with type 1 diabetes. Influence of Helicobacter pylori," Pediatric Diabetes, vol. 10, no. 5, pp. 316-320, 2009.

[69] I. Ciortescu, C. Sfarti, M. Stan, M. Graur, and C. Stanciu, "Prevalence of Helicobacter pylori infection in patients with diabetes mellitus," Revista Medico-Chirurgicala A Societatii de Medici si Naturalisti din Iasi, vol. 113, no. 4, pp. 10481055, 2009.

[70] G. Lazaraki, J. Kountouras, S. Metallidis et al., "Endothelial nitric oxide synthase (eNOS) is not upregulated in gastric mucosa of Helicobacter pylori ( $H$. pylori)-positive patients with type 2 diabetes mellitus," Digestive and Liver Disease, vol. 41, no. 4, pp. 253-262, 2009.

[71] L. Q. Zhang and M. Q. Zhang, "Gastroparesis causing by Helicobacter pylori infection in type 2 diabetes (Chinese)," Chinese Journal of Prevention and Control of Chronic Diseases, vol. 17, no. 4, pp. 413-414, 2009.

[72] X. J. Yu, "The association between $H$. pylori infection and type 2 diabetes (Chinese)," Modern Practical Medicine, vol. 21, no. 5, p. 481, 2009.

[73] K. Ariizumi, T. Koike, S. Ohara et al., "Incidence of reflux esophagitis and H. pylori infection in diabetic patients," World Journal of Gastroenterology, vol. 14, no. 20, pp. 3212-3217, 2008.

[74] M. Demir, H. S. Gokturk, N. A. Ozturk, M. Kulaksizoglu, E. Serin, and U. Yilmaz, "Helicobacter pylori prevalence in diabetes mellitus patients with dyspeptic symptoms and its relationship to glycemic control and late complications," Digestive Diseases and Sciences, vol. 53, no. 10, pp. 2646-2649, 2008.

[75] S. A. Hamed, N. F. Amine, G. M. Galal et al., "Vascular risks and complications in diabetes mellitus: the role of helicobacter pylori infection," Journal of Stroke and Cerebrovascular Diseases, vol. 17, no. 2, pp. 86-94, 2008.

[76] C. Nicholas, E. Ugwuja, B. N. Ejikeme, and N. C. Obeka, "Helicobacter pylori seropositivity in Nigerians with type 2 diabetes mellitus," Internet Journal of Tropical Medicine, vol. 4, no. 2, 2008.

[77] S. Q. Yan, J. F. Zhan, H. N. Tian, W. Tan, and H. Jin, "The association between $H$. pylori infection and type 2 diabetes (Chinese)," Journal of Practical Diabetology, vol. 4, no. 4, pp. 21-22, 2008. 
[78] D. Y. Wang, L. M. Zhou, and H. M. Tong, "Prevalence of Helicobacter pylori infection in type 2 diabetic mellitus patients with complicated peptic ulcer and effect of eradication," Chinese Journal of Nosocomiology, vol. 10, pp. 2191-2193, 2015.

[79] Y. F. Ji, C. F. Zhong, and S. J. Ji, "Clinical analyses on 120 cases of endoscopy in diabetic gastropathy patients (Chinese)," Chinese Journal of Postgraduates of Medicine, vol. 31, no. 16, 2008.

[80] A. Bener, R. Micallef, M. Afifi, M. Derbala, H. M. Al-Mulla, and M. A. Usmani, "Association between type 2 diabetes mellitus and Helicobacter pylori infection," The Turkish Journal of Gastroenterology, vol. 18, no. 4, pp. 225-229, 2007.

[81] G. X. Sun, Y. J. Tan, and Y. L. Zhu, "The relationship between type 2 diabetes mellitus and H. pylori infection," Journal of Practical Medical Techniques, vol. 14, no. 28, pp. 3868-3869, 2007.

[82] S. M. Jaber, "Helicobacter pylori seropositivity in children with chronic disease in Jeddah, Saudi Arabia," Saudi Journal of Gastroenterology, vol. 12, no. 1, pp. 21-26, 2006.

[83] X. M. Lu, L. M. Chen, H. Yang, C. F. Ye, S. G. Yang, and M. Xiang, "Association of ischemic cardiocerebrovascular disease with Helicobacter pylori infection in patients with type 2 diabetes," Chinese Journal of Endocrinology and Metabolism, vol. 22, no. 4, pp. 359-360, 2006.

[84] N. E. Gulcelik, E. Kaya, B. Demirbas et al., "Helicobacter pylori prevalence in diabetic patients and its relationship with dyspepsia and autonomic neuropathy," Journal of Endocrinological Investigation, vol. 28, no. 3, pp. 214-217, 2005.

[85] R. F. Gillum, "Infection with Helicobacter pylori, coronary heart disease, cardiovascular risk factors, and systemic inflammation: the Third National Health and Nutrition Examination Survey," Journal of the National Medical Association, vol. 96, no. 11, pp. 1470-1476, 2004.

[86] R. Anastasios, C. Goritsas, C. Papamihail, R. Trigidou, P. Garzonis, and A. Ferti, "Helicobacter pylori infection in diabetic patients: prevalence and endoscopic findings," European Journal of Internal Medicine, vol. 13, no. 6, p. 376, 2002.

[87] S. Cenerelli, P. Bonazzi, R. Galeazzi et al., "Helicobacter pylori masks differences in homocysteine plasma levels between controls and type 2 diabetic patients," European Journal of Clinical Investigation, vol. 32, no. 3, pp. 158-162, 2002.

[88] C. Colombo, P. A. Tomasi, G. F. Meloni, A. M. Marinaro, A. Ogana, and T. Meloni, "Seroprevalence of Helicobacter pylori in children with type 1 diabetes mellitus in Sardinia," Diabetes, Nutrition \& Metabolism, vol. 15, no. 2, pp. 91-95, 2002.

[89] S. Maule, L. Lombardo, C. Rossi et al., "Helicobacter pylori infection and gastric function in primary autonomic neuropathy," Clinical Autonomic Research, vol. 12, no. 3, pp. 193-196, 2002.

[90] J. Zelenková, A. Soucková, M. Kvapil, A. Soucek, J. Vejvalka, and J. Segethova, "Helicobacter pylori and diabetes mellitus," Casopis lekaru ceskych, vol. 141, no. 18, pp. 575-577, 2002.

[91] G. T. Ko, F. K. Chan, W. B. Chan et al., "Helicobacter pylori infection in Chinese subjects with type 2 diabetes," Endocrine Research, vol. 27, no. 1-2, pp. 171-177, 2001.

[92] A. Ivandić, D. Bozić, B. Dmitrović, A. Vcev, and S. Canecki, "Gastropathy and diarrhea in diabetic patients: the presence of helicobacteriosis and PAS-positive vascular deposits in gastric and duodenal mucosa," Wiener Klinische Wochenschrift, vol. 113, no. 5-6, pp. 199-203, 2001.
[93] M. Ravera, S. Bahenda, R. Owor, and R. Visonà, "Helicobacter pylori infection in diabetic patients with dyspepsia in Uganda," Digestive and Liver Disease, vol. 33, no. 4, pp. 390-391, 2001.

[94] M. Marrollo, G. Latella, D. Melideo et al., "Increased prevalence of Helicobacter pylori in patients with diabetes mellitus," Digestive and Liver Disease, vol. 33, no. 1, pp. 21-29, 2001.

[95] M. Quatrini, V. Boarino, A. Ghidoni, A. R. Baldassarri, P. A. Bianchi, and M. T. Bardella, "Helicobacter pylori prevalence in patients with diabetes and its relationship to dyspeptic symptoms," Journal of Clinical Gastroenterology, vol. 32, no. 3, pp. 215-217, 2001.

[96] A. Vazeou, A. Papadopoulou, I. W. Booth, and C. S. Bartsocas, "Prevalence of gastrointestinal symptoms in children and adolescents with type 1 diabetes," Diabetes Care, vol. 24, no. 5, pp. 962-964, 2001.

[97] H. H. Xia, N. J. Talley, E. P. Kam, L. J. Young, J. Hammer, and M. Horowitz, "Helicobacter pylori infection is not associated with diabetes mellitus, nor with upper gastrointestinal symptoms in diabetes mellitus," The American Journal of Gastroenterology, vol. 96, no. 4, pp. 1039-1046, 2001.

[98] L. J. Zhao, "H. pylori infection and type 2 diabetes (Chinese)," Beijing Medical Journal, vol. 24, no. 1, pp. 60-61, 2002.

[99] D. Arslan, M. Kendirci, S. Kurtoglu, and M. Kula, "Helicobacter pylori infection in children with insulin dependent diabetes mellitus," Journal of Pediatric Endocrinology \& Metabolism, vol. 13, no. 5, pp. 553-556, 2000.

[100] M. P. Dore, M. Bilotta, H. M. Malaty et al., "Diabetes mellitus and Helicobacter pylori infection," Nutrition, vol. 16, no. 6, pp. 407-410, 2000.

[101] N. Güvener, Y. Akcan, I. Paksoy et al., "Helicobacter pylori associated gastric pathology in patients with type II diabetes mellitus and its relationship with gastric emptying: the Ankara study," Experimental and Clinical Endocrinology \& Diabetes, vol. 107, no. 3, pp. 172-176, 1999.

[102] S. Salardi, E. Cacciari, M. Menegatti et al., "Helicobacter pylori and type 1 diabetes mellitus in children," Journal of Pediatric Gastroenterology and Nutrition, vol. 28, no. 3, pp. 307-309, 1999.

[103] D. A. Luisde, H. de la Calle, G. Roy et al., "Helicobacter pylori infection and insulin-dependent diabetes mellitus," Diabetes Research and Clinical Practice, vol. 39, no. 2, pp. 143-146, 1998.

[104] A. Gasbarrini, V. Ojetti, D. Pitocco et al., "Helicobacter pylori infection in patients affected by insulin-dependent diabetes mellitus," European Journal of Gastroenterology \& Hepatology, vol. 10, no. 6, pp. 469-472, 1998.

[105] S. Gentile, S. Turco, B. Oliviero, and R. Torella, "The role of autonomic neuropathy as a risk factor of Helicobacter pylori infection in dyspeptic patients with type 2 diabetes mellitus," Diabetes Research and Clinical Practice, vol. 42, no. 1, pp. 41-48, 1998.

[106] M. Pocecco, E. Buratti, A. Tommasini, G. Torre, and T. Not, "High risk of Helicobacter pylori infection associated with cow's milk antibodies in young diabetics," Acta Paediatrica, vol. 86, no. 7, pp. 700-703, 1997.

[107] M. Małlecki, A. I. Bień, D. Galicka-Latałla, J. Stachura, and J. Sieradzki, "The prevalence of Helicobacter pylori infection and types of gastritis in diabetic patients. The Kraków Study," Experimental and Clinical Endocrinology \& Diabetes, vol. 104, no. 5 , pp. $365-369,1996$. 


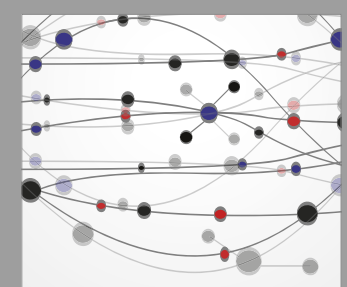

The Scientific World Journal
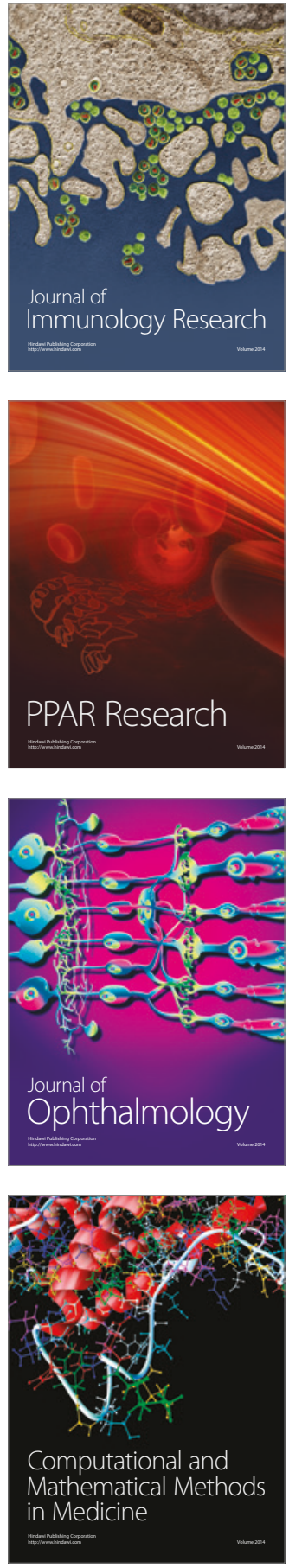

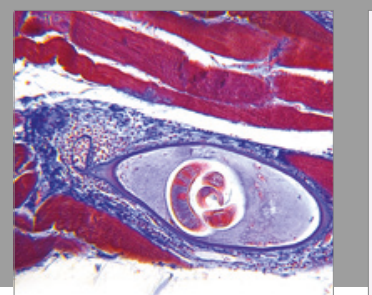

Gastroenterology Research and Practice
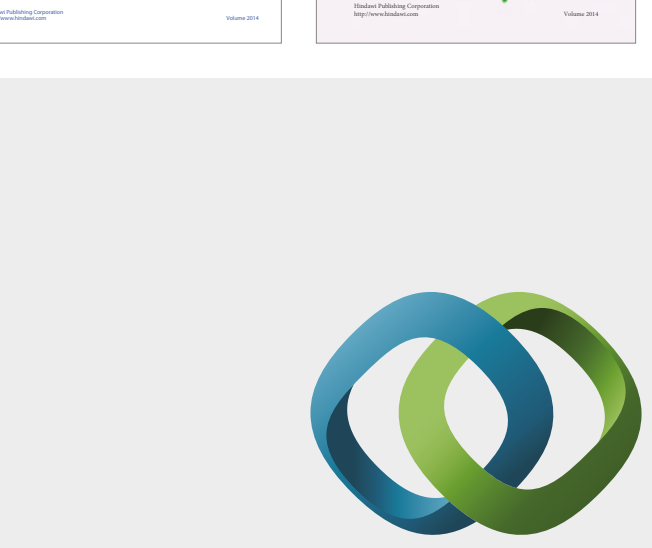

\section{Hindawi}

Submit your manuscripts at

https://www.hindawi.com
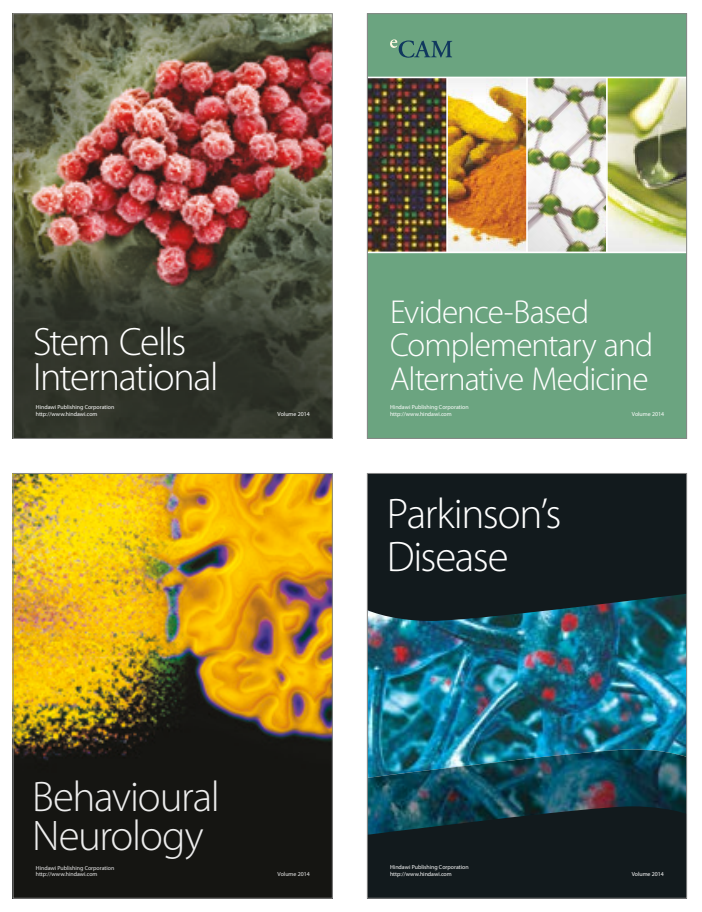
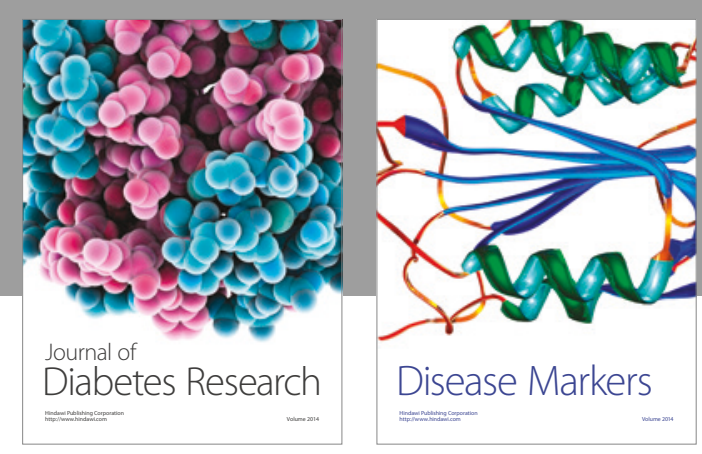

Disease Markers
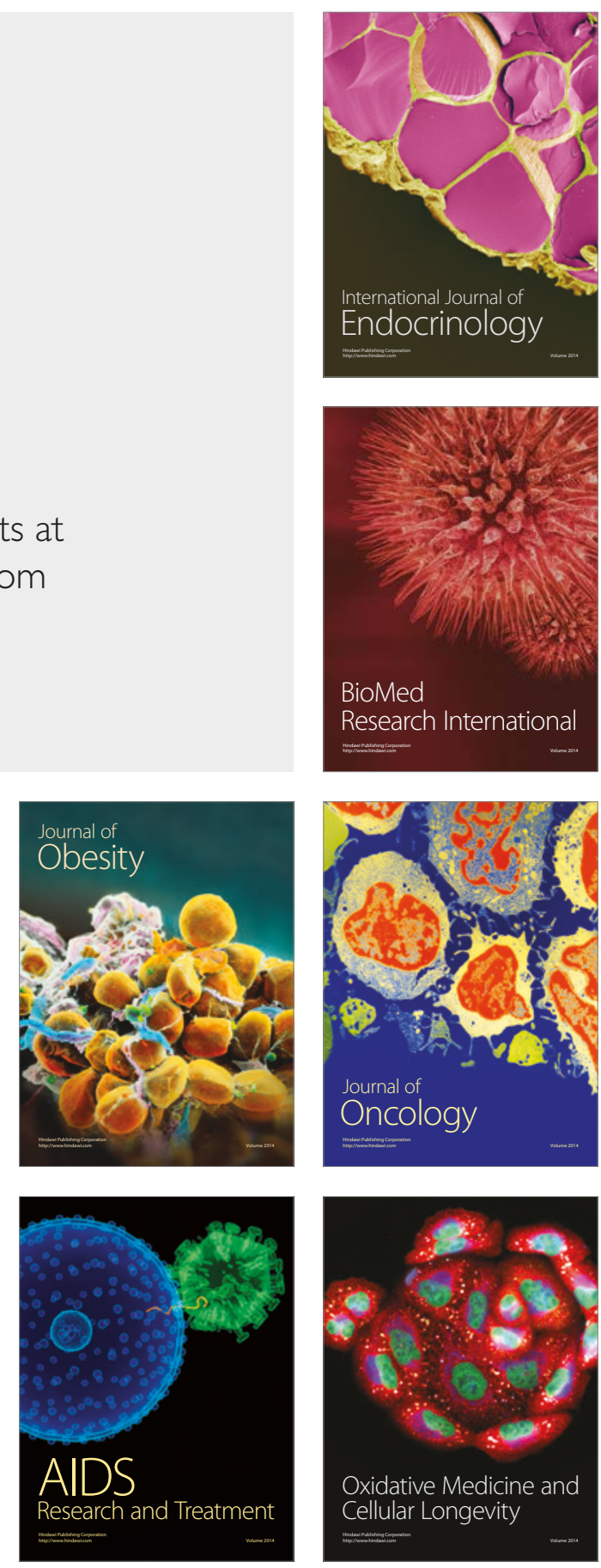